\title{
New Statistical Metrics for Multisite Replication Projects
}

\author{
Maya B. Mathur*1 and Tyler J. VanderWeele ${ }^{2}$ \\ ${ }^{1}$ Quantitative Sciences Unit, Stanford University \\ ${ }^{2}$ Department of Epidemiology, Harvard University
}

${ }^{*}$ Correspondence to: Maya B. Mathur (mmathur@stanford.edu), Quantitative Sciences Unit, 1701 Page Mill Road, Palo Alto, CA, 94304.

\begin{abstract}
Increasingly, researchers are attempting to replicate published original studies using large, multisite replication projects, at least 134 of which are completed or ongoing. These designs are promising to assess whether the original study is statistically consistent with the replications and to re-assess the strength of evidence for the scientific effect of interest. However, existing analyses generally focus on single replications; when applied to multisite designs, they provide an incomplete view of aggregate evidence and can lead to misleading conclusions about replication success. We propose new statistical metrics representing: (1) the probability that the original study's point estimate would be at least as extreme as it actually was, if in fact the original study were statistically consistent with the replications; (2) the estimated proportion of true effects agreeing in direction with the original study. Generalized versions of the second metric allow consideration of only true effects of scientifically meaningful size that agree in direction, or alternatively that disagree in direction, with the original study. These metrics apply when there are at least 10 replications. The first metric assumes normal true effects but appears robust to violations in simulations; the second is distribution-free. We provide $R$ packages (Replicate and MetaUtility).
\end{abstract}

Key words: replication, reproducibility, heterogeneity, effect sizes 


\section{INTRODUCTION}

Several social science disciplines have recently moved to empirically assess replicability of the published literature through systematic, third-party replications. Investigators conducting replications often seek to assess, firstly, how similar the results of the replication studies are to those of the original studies, that is, the extent to which the original studies are statistically consistent or inconsistent with their replications (Anderson \& Maxwell, 2016). Second, investigators often aim to use replications to re-assess evidence strength for the scientific effect under investigation (Anderson \& Maxwell, 2016), ideally while minimizing bias (e.g., through protocol and analysis preregistration and a priori editorial approval (Simons et al. 2014) and while ensuring high statistical power.

Novel designs for reproducibility research now exist to address these objectives with more sophistication than simple designs involving a single replication of an original study. Some high-impact experimental psychology journals now encourage projects in which multiple independent sites attempt to replicate a single, published original study using a standardized experimental protocol closely approximating the original and developed with input from the original authors (Simons et al., 2014). Extensions (sometimes called "Many Labs" projects) select multiple original studies and subject each to a multisite replication (Ebersole et al. (2016); Klein et al. (2014)), and others have applied a similar approach to replicate new original research prior to its publication (Schweinsberg et al., 2016). We use the term "manyto-one design" to refer generically to any design in which an original study is replicated in multiple sites. Many-to-one replication research is a nascent, but rapidly expanding, field: we are aware of at least 79 completed and 55 ongoing many-to-one replication studies to date, all completed or initiated since 2014 and in experimental psychology and experimental philosophy alone (completed: Alogna et al. (2014); Bouwmeester et al. (2017); Cheung et al. (2016); Cova, F., et al. (2018); Ebersole et al. (2016); Eerland et al. (2016); Hagger et al. (2016); Klein et al. (2014); Schweinsberg et al. (2016); Wagenmakers et al. (2016); ongoing: 
Association for Psychological Science (2018); Ebersole, C.R., et al. (2018); Klein, R.A., et al. (2018); Schweinsberg \& Uhlmann (2018)).

However, the adoption of many-to-one designs in the social sciences has outpaced develppment of corresponding statistical analyses. Existing work (Andrews \& Kasy (2017); Etz \& Vandekerckhove (2016); Patil et al. (2016); Simonsohn (2015); Verhagen \& Wagenmakers (2014) has proposed analytic approaches for a single replication of a single study or designs in which numerous original studies across a discipline or domain are each replicated once (here termed "one-to-one designs"), as in Open Science Collaboration (2015) and in Camerer et al. (2016). Other potentially relevant work has not been adapted to the replication context (Gadbury \& Iyer (2000); Gadbury et al. (2001); Heckman et al. (1997); Longford (1999)). However, many-to-one designs pose unique statistical challenges and opportunities. Results of many-to-one replications often suggest effect heterogeneity across sites despite use of standardized protocols (for example, 8 of 16 replications in Klein et al. (2014) suggested "statistically significant" evidence of heterogeneity), yet current analysis approaches do not adequately account for heterogeneity. As we will discuss, this can lead to misleading assessments of consistency between the original study and the replications and of the strength of evidence for the effect under investigation. Additionally, results of many-to-one designs often lead to unresolved debates regarding the extent to which the original study "replicated" or "did not replicate", but these debates remain highly speculative, perhaps partly because few directly relevant quantitative metrics are currently available.

We therefore propose new statistical metrics specifically designed for many-to-one designs. To assess statistical consistency, we provide a metric $\left(P_{\text {orig }}\right)$ representing the probability that the effect estimate from the original study would be as extreme or more extreme than it actually was if, in fact, the original study and the replications were statistically consistent in the sense of being drawn from the same distribution. To assess evidence strength, we provide a metric estimating the proportion of true effects agreeing in direction with the original effect estimate $\left(\widehat{P}_{>0}\right)$. Because replication effects that agree in direction with the 
original, but are very weak, may in fact be considered insufficient evidence to support the original effect, we also demonstrate how to generalize this metric to consider the proportion of true effects that not only agree in direction with the original, but are also stronger than a user-chosen threshold of scientifically meaningful size $\left(\widehat{P}_{>q}\right)$. Lastly, we also provide a counterpart metric estimating the proportion of true effects in the opposite direction of the original $\left(\widehat{P}_{<q^{*}}\right)$. In contrast to existing metrics, the proposed metrics account for all relevant sources of statistical uncertainty in many-to-one replication designs, including heterogeneity Kenny \& Judd (2018), and they harness the specific strengths of many-to-one designs. These metrics are mathematically straightforward, but to the best of our knowledge, have not yet been reported in any published many-to-one replication. We provide $\mathrm{R}$ functions in the packages Replicate and MetaUtility to conduct all proposed analyses.

\section{APPLIED EXAMPLE}

As a running example, we will consider one of several many-to-one replication attempts conducted by Ebersole et al. (2016). Specifically, each of 21 independent labs used a common protocol to replicate a classic psychology experiment (Monin \& Miller (2001)'s Experiment 1) on "moral credentialing" theory, which proposes that people given an initial opportunity to demonstrate that they are not prejudiced (and thus establish "moral credentials") are more likely to display apparently prejudiced attitudes in subsequent tasks (having licensed themselves to do so because of their previously established credentials). In the replicated experiment, the initial task required subjects to agree or disagree with potentially sexist statements. In the initial task, subjects were randomized to a credentialing condition in which the statements described "most women" (e.g., "Most women need a man to protect them") or to a control condition, in which the same statements described only "some women". Thus, credentialing statements were designed to induce higher disagreement than control statements, allowing subjects in the former condition to more clearly establish themselves as non-sexists. The dependent variable was subjects' degree of preference for male candidates 
in an imagined hiring scenario. As predicted, subjects in the credentialing condition more strongly preferred to hire male candidates than did control subjects (corresponding to an effect size of $r=0.21$ on Pearson's correlation scale, (95\% confidence interval [CI]: 0.09, 0.32; $\left.p=7 \cdot 10^{-4}\right)$. Monin \& Miller (2001) also reported an unexpected interaction of credentialing condition with the subject's sex, and Ebersole et al. (2016) attempted to replicate both the main effect and the interaction. For brevity, we focus only on the main effect.

\section{EXISTING METRICS}

We first review metrics commonly reported in many-to-one designs as well as those developed for other designs, but that are frequently reported in many-to-one designs. First, nearly all many-to-one designs report a pooled estimate of the effect size in the replications. The pooled estimate is usually estimated by meta-analyzing effect sizes from the replications or by fitting a mixed model to individual subject data. For example, fitting a random-effects meta-analysis model to Ebersole et al. (2016)'s replication studies on moral credentialing estimates an average effect size of 0.07 (95\% CI: 0.02, 0.12) on the Pearson's correlation scale; both the replicators and the lead author of the original study (Monin, 2016) interpreted this finding as a successful replication supporting moral credentialing. Regardless of modeling approach, this metric estimates the average true effect size across the replications. This is adequate if replications exhibit little heterogeneity but provides an incomplete picture in the presence of heterogeneity across replication studies. Such heterogeneity may occur, for example, if replication studies differ with respect to subjects' demographic characteristics (e.g., age, sex, race, or geographic region) or the setting in which the study is conducted (e.g., time of day, physical setting, etc.). As the proposed metrics will formalize, the forest plot in Figure 1 suggests heuristically that although a group of replication point estimates were clustered around the pooled point estimate, several point estimates were in fact in the direction opposite the original, and several were even larger than the original.

As discussed elsewhere in the context of meta-analyses rather than replications (Mathur 


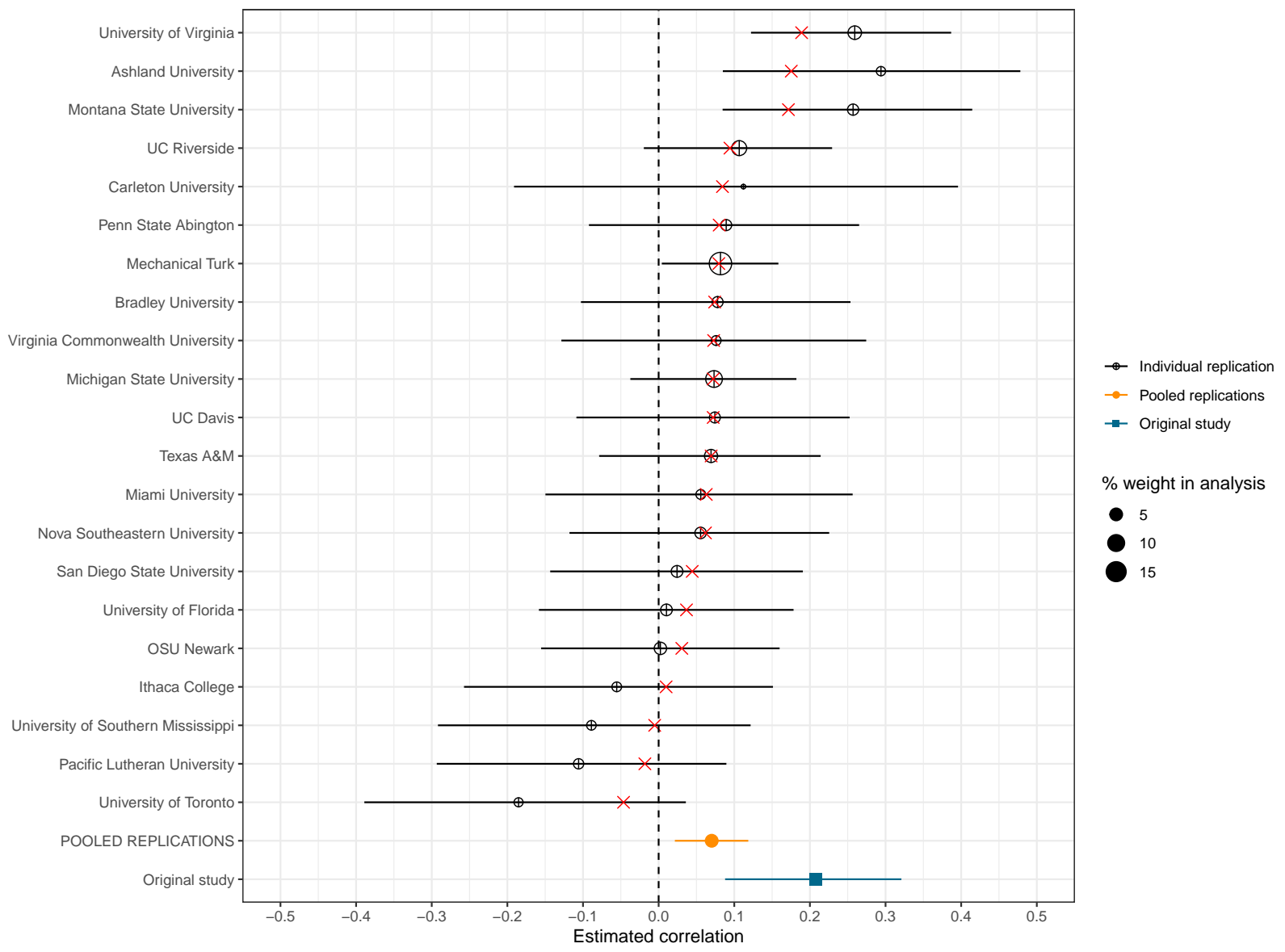

Figure 1: Estimated correlation in each of Ebersole et al. (2016)'s replications (open black circles), ordered by the calibrated estimate of the replication's true effect size (red crosses), discussed in Section 4.2. Circle areas are proportional to the replication's relative inversevariance weight in the meta-analysis. Orange point: meta-analytic pooled estimate across replications. Blue square: estimate in Monin ES Miller (2001)'s original study. Errors bars represent $95 \%$ confidence intervals. 
\& VanderWeele, 2019), under moderate or substantial heterogeneity, a pooled estimate near the null can belie the existence of strong effects in some replication settings. Thus, due to heterogeneity, a many-to-one replication design whose pooled estimate appears not to support the hypothesized effect may nevertheless provide evidence of meaningfully large effects in favor of the original hypothesis in some contexts (for example, locations, subject demographics, variations in protocol administration, etc.). Conversely, if the pooled estimate is in the same direction as the original estimate, but is smaller, we cannot directly discern whether the true effect is never as large as originally reported (and perhaps is too small to warrant scientific interest) or whether it may, in fact, be as large as or larger than the original estimate in some settings. For these reasons, we will recommend supplementing the pooled point estimate with new metrics that additionally characterize heterogeneity.

A widespread metric of statistical consistency assesses whether the replication study obtains a "statistically significant" $p$-value and an effect estimate in the same direction as in the original study (assuming that the original study itself obtained a "significant" $p$-value). This "significance agreement" metric is widely reported in single replications Anderson \& Maxwell, 2016), in one-to-one designs (Camerer et al., 2016, Open Science Collaboration, 2015), and in many-to-one designs. However, as others have noted (Patil et al., 2016, Simonsohn, 2015), "significance agreement" is challenging to interpret because it is a function not only of the nominal $\alpha$-level (e.g., 0.05), but also of power in both the original and the replication study. "Thus, the expected probability of "significance agreement" may be quite low (Andrews \& Kasy, 2017; Patil et al., 2016), though it can be simulated (Patil et al., 2016) or derived (Supplement) for a given original and replication study and then compared to the observed probability. In our running example regarding moral credentialing, $24 \%$ of replications ( 5 of 21) obtained results agreeing in "statistical significance" and effect direction with the original, which appears much lower than the $62 \%$ than we would expect theoretically (based on the original effect size and its standard error, as well as the standard error of each replication). Assessing significance agreement across replication studies is a direct analog to the outdated 
practice of synthesizing evidence across studies in a systematic review by counting the number of studies obtaining "statistically significant" results. The latter "vote-counting" method has extremely poor power, and surprisingly, in some settings the power can decrease as the number of synthesized studies increases (Hedges \& Olkin, 1980).

A variety of more interpretable metrics have been developed for one-to-one replications, and some have also been reported in many-to-one designs. Patil et al. (2016) proposed using the original study to construct a prediction interval representing a plausible range for the effect estimate in the replication study, assuming that the replication and the original study are generated from the same distribution (i.e., they are statistically "consistent"). If indeed the two studies are generated from the same distribution, then regardless of power in either study, there is, by construction, a $95 \%$ probability that the replication effect estimate will fall inside the prediction interval. Simonsohn (2015) proposed a hypothesis test of the replication estimate versus a nonzero null value chosen as the smallest effect size that the original study would have had an estimated $33 \%$ power to detect; this approach can help assess whether the original study was adequately powered to detect the studied effect, but does not directly assess consistency between the original study and the replications nor evidence strength in the replications themselves. Andrews \& Kasy (2017) developed a sophisticated, general statistical model for median-unbiased effect size estimation in one-to-one replication designs such as Open Science Collaboration (2015). Several authors (e.g., Etz \& Vandekerckhove (2016); Verhagen \& Wagenmakers (2014)) recommend using Bayes factors to quantify evidence for and against the null hypothesis.

In a many-to-one design, some of these metrics can be applied individually to each replication study or to the pooled estimate. The former analysis can be informative, but does not aggregate evidence and statistical power across all replications. The latter analysis is subject to the same limitations as the pooled estimate itself, namely that it summarizes a potentially heterogeneous distribution of replication effects by only its mean. In fact, analyses that fail to account for heterogeneity can underestimate (or potentially overestimate) consistency 
when there is in fact heterogeneity, leading to conclusions that can be unduly unfavorable (or potentially unduly favorable) to the original study, as proven in the Supplement.

\section{PROPOSED NEW ANALYSES}

As discussed above, few statistical methods have been developed specifically for many-to-one designs, and those that were developed for other replication designs have limitations when applied to many-to-one designs, particularly in the presence of heterogeneous effects. We therefore propose new metrics to address central objectives of replication research while accounting for all relevant sources of statistical uncertainty, namely statistical error in the original, statistical error in the replication, and heterogeneity. All proposed analyses can be conducted using the R packages Replicate and MetaUtility, described in the Supplement.

\subsection{Consistency of original with replications $\left(P_{\text {orig }}\right)$}

Our first proposed metric assesses statistical consistency. Rather than assuming that the replications and the original measure exactly the same underlying effect size - an assumption implicit to most metrics for single replications - we instead assume that they measure potentially heterogeneous, normally distributed effects. We will then say that the original study is "consistent" with the replications if it is generated from the same underlying distribution as the replications; that is, its true effect size comes from the same distribution as those of the replications. This measure directly accounts for heterogeneity because it compares the original study to the heterogeneous distribution of true effects underlying the replications, rather than to each replication point estimate individually. Then, we define the first proposed metric, called $P_{\text {orig, }}$, as the probability that, if indeed the original is consistent with the replications in this sense, its estimate would be as extreme or more extreme than it actually was. A small value of $P_{\text {orig }}$ would indicate strong evidence that the original study is inconsistent with the replications, whereas a large value would suggest relatively good 
consistency. In practice, if the original study is highly inconsistent with the replications, even accounting for heterogeneity, then we might consider it an anomaly. Future meta-analyses of the published literature might then present analyses both including and excluding such potentially anomalous studies. Additionally, others describe meta-analytically pooling results of an original study with those of a replication (Anderson \& Maxwell, 2016; Open Science Collaboration, 2015); high inconsistency would suggest interpreting such analyses with greater caution.

To estimate $P_{\text {orig }}$, we first define $\widehat{\theta}_{\text {orig }}$ and $\widehat{S E}_{\text {orig }}$ as the point estimate and standard error estimate in the original study, $\widehat{\mu}$ and $\widehat{S E}_{\widehat{\mu}}$ as estimates of the average true effect size in the replications and its standard error, respectively, and $\widehat{\tau}^{2}$ as an estimate of the variance of the true effect sizes across replications. The effect sizes should be estimated on a scale for which the normality assumption is plausible (e.g., the Fisher's $z$ scale), though simulation results suggest robustness to violations of this assumption (Section 6). In practice, $\widehat{\mu}$ and $\widehat{\tau}^{2}$ are most commonly estimated using the pooled estimate and heterogeneity estimate from a random-effects meta-analysis of the replication sites' estimates. Alternatively, they could be estimated by fitting a mixed model to the individual observations themselves (also known as an "individual patient data meta-analysis" (Stewart et al., 2012); both approaches are discussed further in the Supplement. In the main text, for simplicity, we illustrate the common meta-analytic approach, but all analyses can be conducted using any unbiased estimates $\widehat{\mu}$ and $\widehat{\tau}^{2}$ arising from a model with the given distributional assumptions (Supplement).

Then, if the original study is in fact consistent with the replications, the probability that its estimate would be as extreme as we observe it to be is approximately:

$$
P_{\text {orig }}=2 \times\left(1-\Phi\left(\frac{\left|\widehat{\theta}_{\text {orig }}-\widehat{\mu}\right|}{\sqrt{\widehat{\tau}^{2}+\widehat{S E}_{\text {orig }}^{2}+\widehat{S E}_{\widehat{\mu}}^{2}}}\right)\right)
$$

For example, we used the $\mathrm{R}$ package metafor (Viechtbauer, 2010) to fit a random-effects meta-analysis to Ebersole et al. (2016)'s site-level point estimates on the Fisher's $z$ scale via 
restricted maximum likelihood with inverse-variance weights. We thus estimated on the $z$ scale $\widehat{\mu}=0.07, \widehat{S E}_{\widehat{\mu}}=0.02$, and $\widehat{\tau}^{2}=0.003$. We computed $\widehat{\theta}_{\text {orig }}=0.21$ and $\widehat{\mathrm{SE}}_{\text {orig }}=0.06$ for the original study by converting the reported $\eta^{2}$ scale to Fisher's $z$ (Lakens, 2013). Then, using Equation 4.1. we estimated that if the true effect in the original study indeed arose from the same distribution from which the replications were drawn, there would be a $10 \%$ chance that the original effect estimate would be as extreme or more extreme than the observed 0.21 . We can interpret this fairly low, but nonnegligible, probability as being only weakly suggestive of inconsistency. The normality assumption did not appear to be violated based on visual assessment and the Shapiro-Wilk test (Shapiro \& Wilk, 1965), as described below in Section 5 ,

In contrast, previously discussed metrics indicating a low proportion of replications agreeing in "statistical significance" ( $24 \%$ versus $62 \%$ expected) and falling within the original prediction interval (76\% versus $95 \%$ expected) might appear to more strongly suggest inconsistency. These relatively more pessimistic conclusions (compared to the conclusions we might draw from $\left.P_{\text {orig }}\right)$ reflects their failure to account for heterogeneity in the effects across replications. To illustrate quantitatively, we can re-compute $P_{\text {orig, }}$, but this time setting $\widehat{\tau}^{2}=0$ to assume no heterogeneity in the effects across replications. We then obtain a probability of only $4 \%$. This is considerably lower than the $10 \%$ obtained by properly accounting for heterogeneity: a heterogeneous distribution of effects in the replications typically allows a higher chance that any given study would measure a very large or very small effect size (as shown mathematically in the Supplement).

\subsection{Proportion of true effects agreeing in direction with the original $\left(\widehat{P}_{>0}\right)$}

To address a second central objective of replication - re-assessing evidence strength for the scientific effect of interest - we propose a metric $\left(\widehat{P}_{>0}\right)$ to supplement the usual pooled effect estimate and its confidence interval. Unlike these existing metrics, which characterize only the mean of the distribution of true effects in the replications, $\widehat{P}_{>0}$ characterizes both the 
mean and the heterogeneity of this distribution, and it addresses effect size rather than "statistical significance". Specifically, $\widehat{P}_{>0}$ represents the proportion of true effects, among the potentially heterogeneous population from which the replications are a sample, that agree in direction with the original. That is, any nonzero true effect agreeing in direction can be interpreted as a "real" effect supporting the original study's theory (albeit potentially of a smaller effect size). This metric provides additional information beyond that provided by $P_{\text {orig. }}$. That is, $P_{\text {orig }}$ helps assess whether the replications were "successful" in the sense that their results are similar to those of the original study. In contrast, $\widehat{P}_{>0}$ helps assess whether the replications were "successful" in the sense of providing evidence for the effect under investigation, regardless of the results of the original study. We return to this difference in interpretation in analyzing the applied example below as well as in the Conclusion.

To estimate $\widehat{P}_{>0}$, it is not sufficient to simply compute the observed proportion of replication estimates agreeing in direction with the original; such an approach would fail to account for statistical error in the replication estimates. That is, the challenge is to use the distribution of the replication estimates (which has variability due to both heterogeneity and statistical error) to estimate the distribution of true effects (which has variability due only to heterogeneity). As in the context of meta-analysis rather than replication (Mathur \& VanderWeele, in press), the sample proportion can instead be estimated using "calibrated" estimates that have been appropriately shrunk to correct the point estimates' overdispersion due to statistical error (Wang \& Lee, 2019). Let $\widehat{\theta}_{\text {rep }, \mathrm{i}}$ and $\widehat{S E}_{\mathrm{rep}, \mathrm{i}}$ respectively denote the point estimate and standard error estimate in the $i^{\text {th }}$ replication study, and, as before, let $\widehat{\mu}$ and $\widehat{\tau}^{2}$ represent estimates of the mean and variance of the true effects in the replications. Then the calibrated estimate of the true effect in the $i^{t h}$ replication study is Wang \& Lee (2019), Equation (1)):

$$
\widetilde{\theta}_{\text {rep }, \mathrm{i}}=\widehat{\mu}+\sqrt{\widehat{\tau}^{2} /\left(\widehat{\tau}^{2}+\widehat{S E}_{\mathrm{rep}, \mathrm{i}}^{2}\right)}\left(\widehat{\theta}_{\mathrm{rep}, \mathrm{i}}-\widehat{\mu}\right)
$$


Intuitively, the calibrated estimate $\widetilde{\theta}_{\mathrm{rep}, \mathrm{i}}$ shrinks the point estimate $\widehat{\theta}_{\mathrm{rep}, \mathrm{i}}$ toward the estimated mean $\widehat{\mu}$ with a degree of shrinkage that is inversely proportional to the study's precision: relatively imprecise estimates $\widehat{\theta}_{\text {rep,i }}$ (i.e., those with large $\widehat{S E}_{\text {rep,i }}$ ) receive strong shrinkage toward $\widehat{\mu}$, while relatively precise estimates receive less shrinkage and remain closer to their original values ${ }^{a}$. One can then estimate $\widehat{P}_{>0}$ as the sample proportion of calibrated estimates above 0 ; that is, letting $k$ denote the number of replication studies, $\widehat{P}_{>0}=\frac{1}{k} \sum_{i=1}^{k} \mathbb{1}\left\{\widetilde{\theta}_{\text {rep }, \mathrm{i}}>0\right\}$ (Mathur \& VanderWeele, in press). If the point estimates have been transformed for analysis (e.g., from the Pearson's $r$ to the Fisher's $z$ scale), the calibrated estimates will also be on the transformed scale, and the threshold $q$ should therefore be chosen on the transformed scale as well.

For inference, one can bootstrap pairs of $\left(\widehat{\theta}_{\text {rep }, \mathrm{i}}, \widehat{S E}_{\mathrm{rep}, \mathrm{i}}\right)$ by drawing with replacement from the original sample and estimating in turn $\widehat{\mu}$ and $\widehat{\tau}^{2}, \widetilde{\theta}_{\text {rep,i }}$ for each replication, and finally $\widehat{P}_{>0}$ (Mathur \& VanderWeele, in press). A bias-corrected and accelerated (BCa) confidence interval (Carpenter \& Bithell, 2000; Efron, 1987) can then be constructed from the bootstrapped values of $\widehat{P}_{>q}$.

\subsection{Proportion of true effects of scientifically meaningful size $\left(\widehat{P}_{>q}\right.$ and $\left.\widehat{P}_{<q^{*}}\right)$}

The aforementioned $\widehat{P}_{>0}$ treats all effects that agree in direction with the original estimate, even those that are very close to the null, as evidence in favor of the scientific effect under investigation. This is generous toward the original study, and therefore might serve as a useful default analysis. Alternatively, as a more stringent measure of evidence strength, it can also be useful to consider a generalized metric $\left(\widehat{P}_{>q}\right)$ representing the proportion of effects stronger than a non-null threshold, $q$. This approach is similar to equivalence testing and

${ }^{a}$ Readers familiar with empirical Bayes estimation will note that the coefficient $\sqrt{\widehat{\tau}^{2} /\left(\widehat{\tau}^{2}+\widehat{S E}_{\mathrm{rep}, \mathrm{i}}^{2}\right)}$ imposes less shrinkage than the coefficient of $\widehat{\tau}^{2} /\left(\widehat{\tau}^{2}+\widehat{S E}_{\text {rep }, \mathrm{i}}^{2}\right)$ that would be used in the empirical Bayes estimate. By construction, the latter estimates minimize componentwise losses, but produce calibrated estimates that are underdispersed compared to the true effects (Louis, 1984). The present coefficient of $\sqrt{\widehat{\tau}^{2} /\left(\widehat{\tau}^{2}+\widehat{S E}_{\text {rep,i }}^{2}\right)}$ minimizes the distance between the empirical cumulative distribution functions of the calibrated estimates and of the true effects (Louis, 1984), which is the relevant loss function for estimation of $\widehat{P}_{>q}$ and $\widehat{P}_{<q^{*}}$. 
minimal effects testing, which compare a point estimate to null values other than 0 (Lakens et al. 2018). An extensive interdisciplinary literature has provided recommendations on how to choose thresholds for scientifically meaningful effect sizes, which we summarize briefly in the Supplement. For example, suppose that through comparison to well-established effects on similar dependent variables (Supplement), one selects a threshold at a effect size of Cohen's $d=0.20$, or equivalently, an approximate correlation of $r=0.10\left(\right.$ Cohen, 1977). If $\widehat{P}_{>q}$ is large (e.g., 85\%), this suggests that, when drawing from the population distribution of effect sizes underlying the replications, a high proportion of true effects are large enough to warrant scientific interest (e.g., larger than Cohen's $d=0.20$ ). We might therefore conclude that the replications provide strong evidence that the scientific effect of interest is meaningfully strong in many settings. In contrast, if $\widehat{P}_{>q}$ is small, we might instead conclude that the replications fail to support scientifically meaningful effects in most contexts.

Conversely, it can also be useful to consider effects in the direction opposite the original estimate using a second threshold-based metric, $\widehat{P}_{<q^{*}}$. That is, one could select a second threshold representing a scientifically meaningful effect size in the opposite direction (e.g., Cohen's $d=-0.20)$ and estimating the proportion of true effects below this threshold. If the pooled estimate is fairly close to the null or if heterogeneity is substantial, this probability may be nonneglible, suggesting that the experimental manipulation may (perhaps unexpectedly) induce meaningful effects in the opposite direction in some replication settings. Such a finding may help stimulate hypotheses regarding important moderators or boundary conditions on the effect of interest. Additionally, effects in the opposite direction from theoretical predictions may actively support competing theories. Indeed, when evaluating competing theories, researchers sometimes deliberately design experimental manipulations that are expected to induce opposing effects under each candidate theory. Returning to moral credentialing, the theory under investigation predicts that credentialing opportunities would increase subsequent attitudes consistent with prejudice; however, other theories suggest that credentialing opportunities might sometimes decrease such attitudes by prompting 
self-consistency or by priming personal values that discourage prejudice (Monin \& Miller, 2001). Using $\widehat{P}_{<q^{*}}$ to explicitly characterize effects in the opposite direction (rather than simply allowing them to dilute the pooled estimate without additional consideration) may help identify situations, possibly supported by alternative theories, in which such competing effects occur.

These threshold-based metrics are particularly informative when the pooled estimate in the replications is smaller than that of the original study, as is often the case (e.g., Ebersole et al. (2016)). The proportion of true effects above a threshold $\left(\widehat{P}_{>q}\right)$ may then help identify whether: (1) the true effects are closely clustered around a small average effect size, providing little evidence for effects of scientifically meaningful magnitude; versus (2) the true effects are quite variable around a small average effect size, such that there is in fact compelling evidence that effects of scientifically meaningful magnitude occur in some settings (and thus suggesting the importance of examining possible moderators). For example, suppose the original study estimates an effect size of $d=0.85$, but the replications estimate a much smaller pooled effect size of $d=0.40$. Exclusive focus on the existing metrics may then mislead us into considering the replication effort to have succeeded completely (if the pooled point estimate is also "statistically significant") or to have failed completely (if the pooled point estimate is not "statistically significant"). However, if we additionally choose a threshold of scientific importance at, for example, $d=0.20$ and estimate a reasonably high percent (e.g., $75 \%$ ) of true effects exceeding this threshold, then we might instead consider the replications to provide moderately strong evidence for meaningful effect sizes in some replication settings, warranting an assessment of possible moderators. In contrast, if we instead find that only, for example, $8 \%$ of true effects exceed $d=0.20$, then we might instead conclude that the replications provide little evidence to support scientifically meaningful effect sizes (even if the pooled point estimate is "statistically significant"). Similarly to $\widehat{P}_{>0}$, these metrics can be estimated using the appropriate sample proportions of calibrated estimates. That is, letting $q$ be a chosen effect size threshold of scientific importance, $\widehat{P}_{>q}=\frac{1}{k} \sum_{i=1}^{k} \mathbb{1}\left\{\widetilde{\theta}_{\mathrm{rep}, \mathrm{i}}>q\right\}$ and 
$\widehat{P}_{<q^{*}}=\frac{1}{k} \sum_{i=1}^{k} \mathbb{1}\left\{\widetilde{\theta}_{\text {rep }, \mathrm{i}}<q^{*}\right\}$ (Mathur \& VanderWeele, in press). Confidence intervals can again be obtained via BCa bootstrapping.

\subsection{Proportion of replication effects supporting moral credentialing}

In the moral credentialing example, the original study estimated an effect size of $0.21(95 \%$ CI: $0.09,0.32)$ on the Pearson's correlation scale, whereas our meta-analysis of Ebersole et al. (2016)'s replications estimates a pooled effect size of 0.07 (95\% CI: 0.02, 0.12) with estimated heterogeneity $\widehat{\tau}^{2}=0.003$. As discussed previously, the "statistically significant" result in the replications might appear to suggest that the replication effort was successful. But does the small pooled estimate in the replications, despite its "statistical significance", correspond to a high proportion of replication effects supporting credentialing theory? First, we can use $\widehat{P}_{>0}$ to estimate the proportion of true effects above 0 (91\% with $95 \%$ CI: $57 \%, 100 \%$ ). Alternatively, to choose a threshold representing a minimum effect size of scientific importance, we might consider the effect sizes of well-established interventions on the same outcome measure, namely prejudice (see Supplement for more details on choosing a threshold). For example, a metaanalysis of the enormous literature on intergroup contact and prejudice estimated a pooled effect size of $r=-0.21$ among all study designs and $r=-0.33$ among experimental studies (Pettigrew \& Tropp, 2008). We might treat experimental intergroup-contact interventions as a "gold standard" representing the effect sizes on prejudice that are achievable through purposefully designed interventions. In contrast, the proposed moral credentialing effect is not a designed intervention on prejudice but rather a specific, potentially more subtle, cognitive mechanism of prejudice. Thus, to select an effect size threshold for moral credentialing, we might somewhat reduce the magnitude of the gold-standard interventions to, for example, $|r|=0.10$, which corresponds to Fisher's $z \approx 0.10$ on the analysis scale.

Then we can estimate the sample proportion of calibrated estimates above 0.10 as $\widehat{P}_{>q}=\frac{1}{k} \sum_{i=1}^{k} \mathbb{1}\left\{\widetilde{\theta}_{\mathrm{rep}, \mathrm{i}}>0.10\right\}$. This analysis suggests that a minority of effects $(14 \%$ with $95 \%$ CI: $0 \%, 43 \%)$ surpass $r=0.10$. We can also estimate $\widehat{P}_{<q^{*}}$, that is, the proportion of 
true effects of scientifically meaningful magnitude in the direction opposite Monin \& Miller (2001)'s original findings. We might choose a conservative second threshold at, for example, $r=0(z=0$ on the analysis scale) and use the sample proportion of calibrated estimates below $0, \frac{1}{k} \sum_{i=1}^{k} \mathbb{1}\left\{\widetilde{\theta}_{\text {rep }, \mathrm{i}}<0\right\}$, to estimate that few effects $(14 \%$ with $95 \%$ CI: $0 \%, 43 \%)$ are negative ${ }^{b}$

Ultimately, although these replications produce a "statistically significant" point estimate in the same direction as the original study's estimate, we might nevertheless caution that they provide little evidence for effect sizes of comparable strength to the original estimate across replication settings. In the distribution of true effects, there is a high proportion of a nonzero effects in the direction of the original estimate, but most of these effects are considerably smaller than the original estimate. Considering these results along with the previously discussed consistency metric $\left(P_{\text {orig }}=10 \%\right)$, we might say, overall, that the moral credentialing main effect "replicated" in the sense that there is not compelling evidence for inconsistency between the original study and replications (once we account for heterogeneity), yet evidence strength for scientifically meaningful effect sizes of moral credentialing is considerably weaker than suggested by the original study. These complementary findings further illustrate the conceptual distinction between statistical consistency and evidence strength for scientifically meaningful effects of interest.

\section{REPORTING RECOMMENDATIONS AND STATISTICAL DIAGNOSTICS}

\subsection{Calibrated forest plot}

To supplement the proposed statistical metrics, we suggest creating a forest plot displaying the point estimates and $95 \%$ confidence intervals for each replication study, for all replication studies combined, and for the original study (e.g., Figure 1). It may be informative to

\footnotetext{
${ }^{b}$ For this particular example, the point estimates and confidence intervals are the same for $\widehat{P}_{>0.10}$ and $\widehat{P}_{<0}$ because in each case, exactly $3(14 \%)$ of the calibrated estimates were more extreme than the chosen threshold, as can be seen in Figure1.
} 
additionally indicate the calibrated estimate $\widetilde{\theta}_{\text {rep,i }}$ in each replication study (e.g., the red crosses in Figure 1) and to order the replication studies by their calibrated estimates. The calibrated estimate in each replication can serve as an estimate of that replication study's true effect size upon accounting for the study's precision along with information provided by the other replications. Taken together, the calibrated estimates can provide a heuristic sense of the distribution and variability of the true effect sizes themselves, rather than of the potentially noisy point estimates. Furthermore, if there are sites with apparently extreme point estimates or calibrated estimates, one might conduct sensitivity analyses in which the proposed metrics, as well as the diagnostics described below, are re-calculated after excluding these potential outliers.

\subsection{Diagnostics for distributional assumptions}

Our proposed metrics assume that the point estimates are normally distributed around their corresponding true effects, which is reasonable when the replication studies have reasonably large sample sizes and when their estimates are transformed to an appropriate scale for analysis (Sutton et al., 2000). Further, the metric $P_{\text {orig }}$ assumes that the true effect sizes themselves in the replication studies are normally distributed, though the metrics $\widehat{P}_{>0}, \widehat{P}_{>q}$, and $\widehat{P}_{<q^{*}}$ do not use this assumption. In most many-to-one designs, which use mixed modelling or parametric random effects meta-analysis to estimate the pooled effect, this assumption is already implicit. Nevertheless, we would recommend assessing its plausibility as follows. If the normality assumption holds, then the standardized estimates in the replication studies, $\left(\widehat{\theta}_{\text {rep }, \mathrm{i}}-\widehat{\mu}\right) / \sqrt{\widehat{\tau}^{2}+\widehat{S E}_{\mathrm{rep}, \mathrm{i}}^{2}}$, will be approximately normal (Hardy \& Thompson, 1998). Thus, one could test for normality of the standardized estimates via the Shapiro-Francia or ShapiroWilk test, though power may be limited when the number of replication studies is small (Shapiro \& Francia, 1972; Shapiro \& Wilk, 1965). Additionally, sensitivity analyses could be conducted by meta-analyzing the replication study estimates under distribution-free approaches (Fisher \& Tipton, 2015, Hedges et al. 2010) or under more flexible distributional 
assumptions (see Higgins et al. (2009) for a review). If the robustly estimated $\widehat{\mu}$ and $\widehat{\tau}^{2}$ differ substantially from those obtained through parametric meta-analysis, this suggests that the normality assumption may be violated. Because assessment of normality and accurate heterogeneity estimation are challenging with small sample sizes (Hardy \& Thompson, 1998 Veroniki et al. 2016), these metrics should generally be applied only when there are at least 10 replication studies. To the best of our knowledge, this was true in each of the 79 completed many-to-one designs discussed in the Introduction. An exception to this rule of thumb is when there is no heterogeneity, as discussed in the following section.

\subsection{Diagnostics for uncertainty in $P_{\text {orig }}$}

Additionally, estimation uncertainty in $\widehat{\tau}^{2}$ may be considerable when the number of replications is small (Veroniki et al., 2016); it is then possible that many values of $\tau^{2}$ are plausible given the data, resulting in considerable uncertainty about $P_{\text {orig }}$ itself. Three types of diagnostic plots may help identify such situations (c.f. Rubin (1981)). First, one could plot the marginal likelihood of $\tau^{2}$ for a range of values of $\tau_{*}^{2}$, including values both smaller than and larger than the actual point estimate $\widehat{\tau}^{2}$. In practice, to display the likelihood on an interpretable scale, we suggest plotting the marginal likelihood ratio of the hypothetical values $\tau_{*}^{2}$ versus the actual estimate $\widehat{\tau}^{2}$ (Figures 2a and 2b), for example using the R function Replicate: : t2_lkl. Ideally, the peak of the likelihood ratio curve would span a narrow ranges of values around the actual estimate $\widehat{\tau}^{2}$, declining sharply on either side of $\widehat{\tau}^{2}$; this would indicate relatively good certainty about the true value of $\tau^{2}$. If instead the likelihood ratio spans a wide range of values, remaining close to 1 even for values $\tau_{*}^{2}$ that are several-fold smaller or larger than the actual estimate $\widehat{\tau}^{2}$, this suggests that there may be considerable uncertainty in $P_{\text {orig }}$ itself. For example, Figures 2a and Figure 2b display this likelihood ratio for all 21 moral credentialing replications and for only the first three replications, respectively. Because the 21 replications provide more information about the amount of heterogeneity than do only the first three, the likelihood curve is much more peaked in Figure 2a than in Figure 2b, 
and in the latter figure, a wide range of heterogeneity values appear plausible given the very limited data. A plot similar to Figure $2 \mathrm{~b}$ would suggest that the replication data might not be sufficiently informative to assess consistency via $P_{\text {orig. As second and third diagnostic }}$ plots, one could similarly plot a range of values $\tau_{*}^{2}$ versus the ratio $\widehat{\mu} / \tau_{*}^{2}$, which plays a central role in the calculation of $P_{\text {orig, }}$, and also versus $P_{\text {orig }}$ itself (Figures $2 \mathrm{c}$ and $2 \mathrm{~d}$ ). These plots could help indicate how much $P_{\text {orig }}$ would change if a different heterogeneity estimate had been obtained. If, for example, there are hypothetical values $\tau_{*}^{2}$ for which the likelihood ratio is high (i.e., close to 1 based on plots such as Figures $2 \mathrm{a}$ and $2 \mathrm{~b}$ but for which $\widehat{\mu} / \tau_{*}^{2}$ and $P_{\text {orig }}$ differ substantially from their actual estimates, this again suggests that there may be much uncertainty associated with $P_{\text {orig }}$ given the replication data.

\subsection{Diagnostics for bias in $\widehat{P}_{>q}$}

As discussed in Section 6 below, the metric $\widehat{P}_{>q}$ and the related proportion metrics can potentially be biased if, for example, there is low heterogeneity, the true proportion (i.e., $\left.E\left[\widehat{P}_{>q}\right]\right)$ is close to 0 or 1 , or if there is limited replication data. A useful diagnostic for potential bias is the mean of the bootstrapped estimates used to construct the confidence interval. The difference between the mean of the bootstrap estimates and the sample estimate $\widehat{P}_{>q}$ is an estimate of the bias of $\widehat{P}_{>q}$ itself (Davison \& Hinkley (1997), Section 2.2.1), so caution is warranted is the mean of the bootstrap estimates differs considerably from $\widehat{P}_{>q}$. The R function MetaUtility: :prop_stronger automatically returns the mean of the bootstrap estimates for this purpose. 


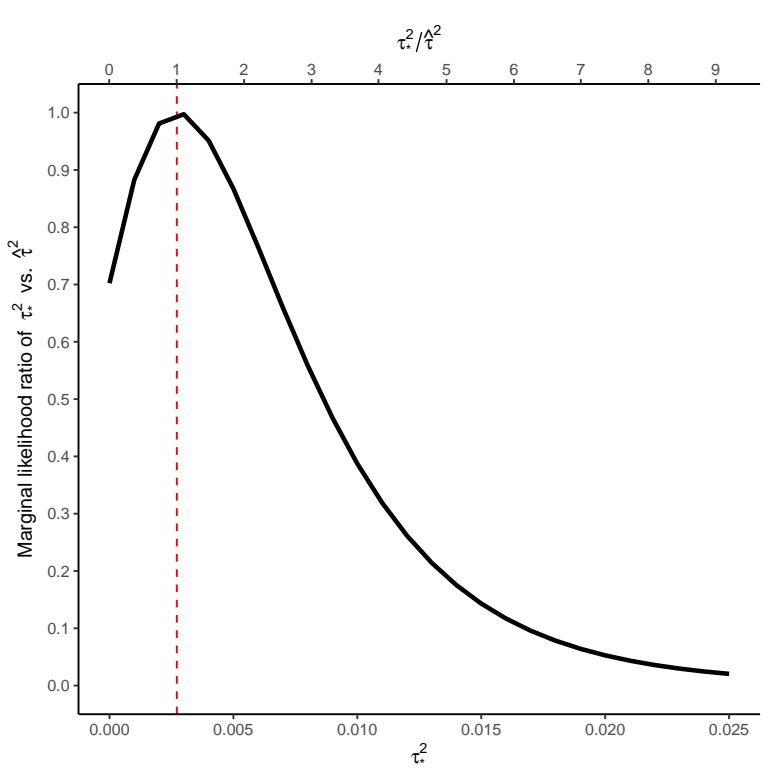

(a)

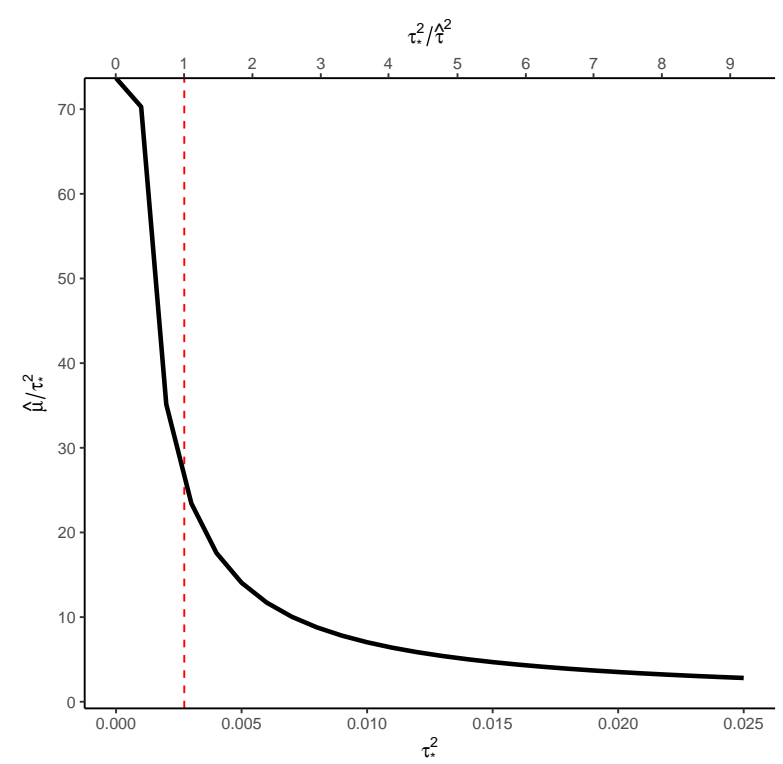

(c)

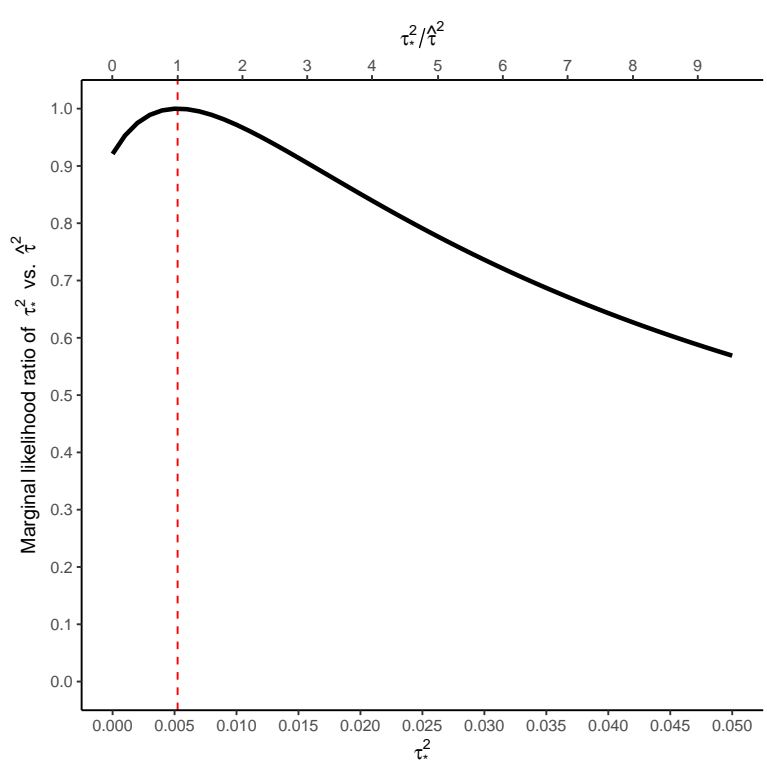

(b)

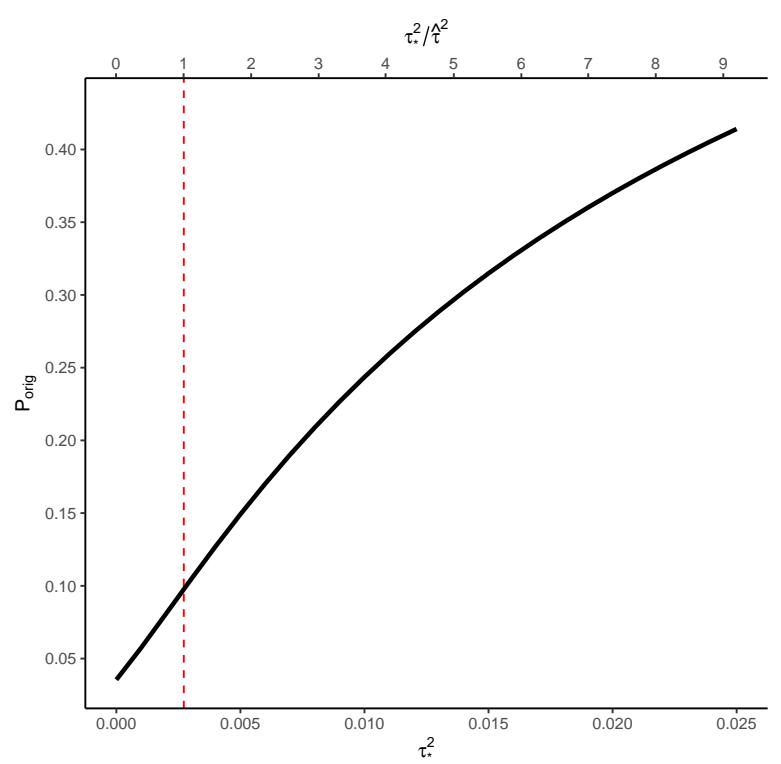

(d)

Figure 2: Diagnostic plots (c.f. Rubin (1981)) relating hypothetical heterogeneity values $\left(\tau_{*}^{2}\right)$ to the marginal likelihood ratio of the hypothetical $\tau_{*}^{2}$ versus the actual restricted maximum likelihood estimate $\widehat{\tau}^{2}$ (Figures 2a and 2b), to the ratio $\widehat{\mu} / \tau_{*}^{2}$ (Figure 2c), and to the resulting $P_{\text {orig }}$ (Figure 2d). Figures 2a, 2c, and 2d were created using the applied example data. Figure 26 was created using only the first three of the moral credentialing replications and serves as a comparison for Panel 2a. Upper x-axis: the ratio of the hypothetical value $\tau_{*}^{2}$ to the actual estimate $\widehat{\tau}^{2}$. Red dashed line: actual estimate $\widehat{\tau}^{2}$. 


\section{Simulation STUdy}

\subsection{Methods}

We conducted a simulation study with two objectives. First, we assessed the Type I error and power of $P_{\text {orig }}$ when it is treated as a level $\alpha=0.05$ test of the hypothesis that the true effect size in the original study is consistent with the replication studies. That is, letting $\mu$ and $\tau^{2}$ denote the unknown true mean and variance of the distribution of effects underlying the replications, we tested $H_{0}: \theta_{\text {orig }} \sim N\left(\mu, \tau^{2}\right)$ vs. $H_{A}: \theta_{\text {orig }} \nsim N\left(\mu, \tau^{2}\right)$, rejecting $H_{0}$ when $P_{\text {orig }}<0.05$. (As described in the Discussion, in practice, we recommend reporting $P_{\text {orig }}$ as a continuous measure rather than relative to a dichotomous $\alpha$ threshold. We use the hypothesis-testing framework for the simulation study merely as a means of benchmarking the metric's performance.) Second, we assessed the bias, root mean square error (RMSE), and confidence interval coverage of $\widehat{P}_{>q}$.

To simulate datasets, we fixed the mean of the true effects to $\mu=0.50$ on the mean difference scale while varying the number of replications $(k)$ between 5 and 25 , the heterogeneity $\tau^{2} \in\{0.002,0.01,0.25\}$, and the sample size in each individual replication $N_{\text {rep }} \in\{50,300\}$. Relevant to the estimation of $P_{\text {orig, }}$, we varied the sample size in the original study $N_{\text {orig }} \in\{30,50,200\}$ and the difference, $\Delta$, between the true effect size in the original study and the true mean in the replications $(\Delta \in\{0,0.20,0.50,1.0\})$. Relevant to the estimation of $\widehat{P}_{>q}$, we chose different thresholds $q$ so that the expectation of $\widehat{P}_{>q}$ varied in $\{0.05,0.10,0.50\}$. In each scenario, we calculated $\widehat{P}_{>q}$ using two different recommended heterogeneity estimators (Veroniki et al. 2016): the restricted maximum likelihood estimator (Raudenbush \& Bryk, 1985) and Paule \& Mandel (1982)'s estimator.

For each of $k$ replications, we generated a true effect, $\theta_{\text {rep }, \mathrm{i}}$, on the raw mean difference scale from a normal distribution, a shifted exponential distribution, a scaled and shifted $t$-distribution with 3 degrees of freedom, or a bimodal uniform mixture distribution. For all distributions, we chose the parameters to provide the desired mean of $\mu=0.50$ and 
heterogeneity $\tau^{2}$. Figure S1 shows true effects simulated from each of the four distributions for each value of $\tau^{2}$. We also generated a true effect for the original study, $\theta_{\text {orig }}$, from a comparable distribution but with mean $\mu+\Delta$. Thus, the null hypothesis regarding $P_{\text {orig }}$ held when $\Delta=0$. For each replication and for the original study, we then simulated subject-level data for a control group with mean 0 and for an intervention group with mean $\theta_{\text {rep, } \mathrm{i}}$ or $\theta_{\text {orig }}$ respectively; each group was of size $N_{\text {rep }} / 2$ or $N_{\text {orig }} / 2$ respectively and had a standard deviation of 1 . Thus, the true within-study standard errors of the estimated mean differences in each replication and in the original study were $\widehat{S E}_{\text {rep } \mathrm{i}}=\sqrt{4 / N_{\text {rep }}}$ and $\widehat{S E}_{\text {orig }}=\sqrt{4 / N_{\text {orig }}}$. We ran scenarios representing all 4,320 possible combinations of the varying parameters, using 5,000 iterates to estimate the confidence interval for $\widehat{P}_{>q}$. We ran at least 1,000 simulation iterates per scenario.

\subsection{Results}

Comprehensive simulation results for all 4,320 scenarios are presented in Figures S2-S8 in the Supplement and can also be explored interactively via a website (https://mmathur .shinyapps.io/RRR_sims/) or downloaded as a dataset (https://osf.io/ufjg4/). The two heterogeneity estimators performed almost identically, so we report here only the results using the restricted maximum likelihood estimator. Across all scenarios with $k \geq 10$ (Supplement), including both normal and non-normal distributions, the average Type I error was $5 \%$. The maximum Type I error rate was $11 \%$, which occurred in scenarios with high heterogeneity as well as few or low-powered replications (e.g., Figure S2). When heterogeneity was low to moderate $\left(\tau^{2}=0.001\right.$ or $\left.\tau^{2}=0.01\right)$, the maximum Type I error rate was $7 \%$ across all sample sizes and numbers of replications and was $6 \%$ when considering only normally distributed true effects. Alternatively, when there was a fairly large number $(k=25)$ of large replication studies $\left(N_{\text {rep }}=300\right)$, the maximum Type I error rate was $7 \%$ across all values of heterogeneity and was $6 \%$ when considering only normally distributed true effects. These results suggest that when $k \geq 10, P_{\text {orig }}$ is rather robust to non-normal true effects, but that 
results should be interpreted cautiously if both: (1) the amount of replication data is limited (due to small sample sizes in the replication studies or to a small number of replication studies) and (2) heterogeneity appears to be high. Table 1 summarizes the results for $P_{\text {orig }}$ for scenarios with $k \geq 10$, since we do not recommend using these methods for $k<10$. For legibility ${ }^{c}$, the table displays results only for scenarios with $N_{\text {orig }}=50$ and $N_{\text {rep }}=300$.

For all scenarios with $k \geq 10$, the bias of $\widehat{P}_{>q}$ was 0.05 on average and was at maximum 0.30. Bias occurred in scenarios with low heterogeneity, an extreme true proportion $\left(E\left[\widehat{P}_{>q}\right]\right)$, and either a small number of replication studies or small sample sizes in the replication studies. When there was a fairly large number $(k=25)$ of large replication studies $\left(N_{\text {rep }}=300\right)$, the average bias was 0.02 , and the maximum bias was 0.12 . Alternatively, when heterogeneity was high $\left(\tau^{2}=0.25\right)$, the maximum bias was 0.07 across all sample sizes and true proportions. The bootstrapping-based inference appeared to compensate for occasional bias in $\widehat{P}_{>q}$, as the minimum coverage across all scenarios with $k \geq 10$ was close to nominal (94\%). Table 2 summarizes the results for $\widehat{P}_{>q}$ for all scenarios with $k \geq 10$. These findings also point to the importance of designing multisite replications with a sufficient number of sites and sufficient sample sizes within each site, and we would encourage researchers designing multisite replication projects to use the interactive simulation results (https://mmathur.shinyapps .io/RRR_sims/) to provide some preliminary guidance.

${ }^{c}$ The means and maxima reported in the text consider each simulation scenario individually and include scenarios with all sample sizes, so differ from the means and maxima listed in aggregated tables. 
Table 1: Type I error ( $\Delta=0$ rows) and power ( $\Delta>0$ rows) of $P_{\text {orig }}$ for scenarios with $k \geq 10$. For legibility, results are shown only for scenarios with with $N_{\text {orig }}=50$ and $N_{\text {rep }}=300$, and statistics aggregate over the other manipulated simulation parameters that are not listed in the column headers.

\begin{tabular}{|c|c|c|c|c|}
\hline Distribution & $\tau^{2}$ & $\Delta$ & Mean rejection rate & Max rejection rate \\
\hline Normal & 0.002 & 0.00 & 0.04 & 0.05 \\
\hline Normal & 0.002 & 0.20 & 0.09 & 0.09 \\
\hline Normal & 0.002 & 0.50 & 0.34 & 0.34 \\
\hline Normal & 0.002 & 1.00 & 0.89 & 0.90 \\
\hline Normal & 0.010 & 0.00 & 0.05 & 0.05 \\
\hline Normal & 0.010 & 0.20 & 0.09 & 0.09 \\
\hline Normal & 0.010 & 0.50 & 0.33 & 0.33 \\
\hline Normal & 0.010 & 1.00 & 0.86 & 0.87 \\
\hline Normal & 0.250 & 0.00 & 0.06 & 0.07 \\
\hline Normal & 0.250 & 0.20 & 0.07 & 0.08 \\
\hline Normal & 0.250 & 0.50 & 0.13 & 0.15 \\
\hline Normal & 0.250 & 1.00 & 0.37 & 0.39 \\
\hline Exponential & 0.002 & 0.00 & 0.05 & 0.05 \\
\hline Exponential & 0.002 & 0.20 & 0.09 & 0.10 \\
\hline Exponential & 0.002 & 0.50 & 0.34 & 0.35 \\
\hline Exponential & 0.002 & 1.00 & 0.89 & 0.90 \\
\hline Exponential & 0.010 & 0.00 & 0.05 & 0.05 \\
\hline Exponential & 0.010 & 0.20 & 0.09 & 0.09 \\
\hline Exponential & 0.010 & 0.50 & 0.31 & 0.32 \\
\hline Exponential & 0.010 & 1.00 & 0.85 & 0.86 \\
\hline Exponential & 0.250 & 0.00 & 0.06 & 0.07 \\
\hline Exponential & 0.250 & 0.20 & 0.08 & 0.09 \\
\hline Exponential & 0.250 & 0.50 & 0.16 & 0.18 \\
\hline Exponential & 0.250 & 1.00 & 0.37 & 0.38 \\
\hline $\mathrm{t}$ & 0.002 & 0.00 & 0.05 & 0.05 \\
\hline $\mathrm{t}$ & 0.002 & 0.20 & 0.09 & 0.10 \\
\hline $\mathrm{t}$ & 0.002 & 0.50 & 0.33 & 0.34 \\
\hline $\mathrm{t}$ & 0.002 & 1.00 & 0.88 & 0.89 \\
\hline $\mathrm{t}$ & 0.010 & 0.00 & 0.05 & 0.06 \\
\hline $\mathrm{t}$ & 0.010 & 0.20 & 0.09 & 0.09 \\
\hline $\mathrm{t}$ & 0.010 & 0.50 & 0.28 & 0.30 \\
\hline $\mathrm{t}$ & 0.010 & 1.00 & 0.80 & 0.81 \\
\hline $\mathrm{t}$ & 0.250 & 0.00 & 0.07 & 0.08 \\
\hline
\end{tabular}




\begin{tabular}{ccccc}
$\mathrm{t}$ & 0.250 & 0.20 & 0.08 & 0.09 \\
$\mathrm{t}$ & 0.250 & 0.50 & 0.11 & 0.12 \\
$\mathrm{t}$ & 0.250 & 1.00 & 0.23 & 0.24 \\
\hline Uniform mixture & 0.002 & 0.00 & 0.04 & 0.04 \\
Uniform mixture & 0.002 & 0.20 & 0.09 & 0.09 \\
Uniform mixture & 0.002 & 0.50 & 0.34 & 0.35 \\
Uniform mixture & 0.002 & 1.00 & 0.89 & 0.89 \\
Uniform mixture & 0.010 & 0.00 & 0.05 & 0.05 \\
Uniform mixture & 0.010 & 0.20 & 0.09 & 0.09 \\
Uniform mixture & 0.010 & 0.50 & 0.32 & 0.34 \\
Uniform mixture & 0.010 & 1.00 & 0.86 & 0.87 \\
Uniform mixture & 0.250 & 0.00 & 0.03 & 0.04 \\
Uniform mixture & 0.250 & 0.20 & 0.05 & 0.06 \\
Uniform mixture & 0.250 & 0.50 & 0.14 & 0.15 \\
Uniform mixture & 0.250 & 1.00 & 0.40 & 0.42 \\
\hline
\end{tabular}


Table 2: Bias and RMSE of $\widehat{P}_{>q}$ for scenarios with $k \geq 10$. Statistics aggregate over the other manipulated simulation parameters that are not listed in the column headers.

\begin{tabular}{ccccc}
\hline Distribution & $\tau^{2}$ & $N_{\text {rep }}$ & Mean bias & Mean RMSE \\
\hline Normal & 0.002 & 50 & 0.15 & 0.29 \\
Normal & 0.002 & 300 & 0.07 & 0.19 \\
Normal & 0.010 & 50 & 0.06 & 0.20 \\
Normal & 0.010 & 300 & 0.01 & 0.12 \\
Normal & 0.250 & 50 & -0.00 & 0.10 \\
Normal & 0.250 & 300 & -0.00 & 0.09 \\
\hline Exponential & 0.002 & 50 & 0.15 & 0.28 \\
Exponential & 0.002 & 300 & 0.08 & 0.18 \\
Exponential & 0.010 & 50 & 0.08 & 0.19 \\
Exponential & 0.010 & 300 & 0.04 & 0.13 \\
Exponential & 0.250 & 50 & 0.02 & 0.10 \\
Exponential & 0.250 & 300 & 0.00 & 0.09 \\
\hline $\mathrm{t}$ & 0.002 & 50 & 0.12 & 0.26 \\
$\mathrm{t}$ & 0.002 & 300 & 0.04 & 0.17 \\
$\mathrm{t}$ & 0.010 & 50 & 0.04 & 0.17 \\
$\mathrm{t}$ & 0.010 & 300 & 0.01 & 0.11 \\
$\mathrm{t}$ & 0.250 & 50 & -0.00 & 0.09 \\
$\mathrm{t}$ & 0.250 & 300 & -0.00 & 0.09 \\
\hline Uniform mixture & 0.010 & 50 & 0.08 & 0.30 \\
Uniform mixture & 0.010 & 300 & 0.03 & 0.21 \\
\hline Uniform mixture & 0.002 & 50 & 0.16 & 0.14 \\
\hline Uniform mixture & 0.002 & 300 & 0.08 &
\end{tabular}

We have primarily discussed our metrics in the context of many-to-one designs conducted under a shared replication protocol and in which true effects are heterogeneous. Here, we discuss other designs and settings to which the proposed metrics apply, potentially with modified interpretations. 


\subsection{Replications with no apparent heterogeneity}

If a random-effects meta-analysis or similar individual subject-level mixed model yields a negligible statistical estimate of heterogeneity $\left(\widehat{\tau}^{2} \approx 0\right)$ with a confidence interval including only small values, then $\widehat{\mu}$ can be interpreted as an estimate of the single true effect size underlying all replication studies and will approximately coincide with the corresponding estimate from a fixed-effects meta-analysis or linear regression (Rice et al., 2018, Riley et al., 2011). Because heterogeneity estimators can be highly variable when the number of studies is small (Veroniki et al., 2016), it is important to report the confidence interval for $\tau^{2}$ when adopting this "fixed-effects" approach. Under this framework, $P_{\text {orig }}$ can still be informative to assess consistency and is interpretable as the probability that the original study's point estimate would be at least as extreme as that observed if the original study had unbiasedly measured the same true effect as the replication studies. Without heterogeneity, $P_{\text {orig }}$ does not require a normality assumption and can be reported with as few as one replication study, and it becomes a continuous counterpart to a prediction interval in which all replication data are analyzed in aggregate, without regard to site (Supplement). The metrics $\widehat{P}_{>0}, \widehat{P}_{>q}$, and $\widehat{P}_{<q^{*}}$ are no longer relevant because all true effects are estimated to be identical.

\subsection{Single replications and one-to-one replications}

Single replications or one-to-one replication projects preclude estimating heterogeneity for any given replication study, and existing analysis approaches implicitly assume no heterogeneity. If such an assumption is reasonable, then $P_{\text {orig }}$ can be computed with $\widehat{\tau}^{2}=0$, retaining the same interpretation as above; of course, such analyses would need to be interpreted with caution because the assumption of no heterogeneity is not testable in these designs. 


\section{3. "Many Labs" designs}

In designs in which multiple original studies are each replicated in many sites (Ebersole et al. 2016; Klein et al. 2014, Schweinsberg et al., 2016), the proposed metrics permit direct comparison or aggregation of results across many-to-one replications of multiple original studies. For example, one could estimate the proposed metrics for each original study and report the average consistency $\left(P_{\text {orig }}\right)$ as a global summary measure of replication success. The average $\widehat{P}_{>0}$ could also be reported as a global summary of replication evidence strength across numerous scientific effects.

\subsection{Conceptual replications}

We have so far considered contexts in which all replications share a single protocol closely approximating that of the original study (sometimes called "direct replications"). However, some researchers question using only direct replications in many-to-one designs, arguing that these designs assess replicability of a specific operationalization of a theory, rather than of the theory itself (Baumeister \& Vohs, 2016). Others advocate supplementing direct replications with "conceptual replications" that assess the same theory as the original study, but using a different operationalization (Crandall \& Sherman (2016); Lynch et al. (2015); Monin et al. (2014); see also dissent by Nosek et al. (2012); Simons (2014)). For example, replication sites in a conceptual many-to-one design could implement different experimental protocols, each approved by the original authors. Conceptual replications create heterogeneity by design, which exacerbates problems with the metrics proposed prior to this paper (e.g., leading to particularly unfavorable assessments of consistency and inadequately characterizing evidence strength). In contrast, our proposed metrics could simply be applied without modification as they take into account heterogeneity across replications. They would retain their original interpretations, but $\widehat{P}_{>0}$ could then additionally be interpreted as the probability that a new operationalization of the theory at stake would yield a true effect size either in the 
same direction as the theoretical prediction. Such an interpretation holds only when the new operationalization under consideration can be treated as comparable to the range of protocols considered in the conceptual replications.

\section{Discussion}

We have proposed intuitively tractable metrics (implemented in the $\mathrm{R}$ packages Replicate and MetaUtility) for statistical consistency between the original study and replications and for evidence strength in many-to-one replication designs with potential heterogeneity. Such replication projects could report the new metric $P_{\text {orig }}$ to convey consistency and could report the usual pooled estimate $(\widehat{\mu})$, heterogeneity estimate $\left(\widehat{\tau}^{2}\right)$, plus $\widehat{P}_{>0}$ (and possibly also $\widehat{P}_{>q}$ and $\widehat{P}_{<q^{*}}$ ) to re-assess evidence strength for the scientific effect of interest. The proposed metrics account for all relevant sources of statistical uncertainty and can therefore yield different conclusions from existing metrics when the replications are heterogeneous. These metrics can also help identify situations in which there is good statistical consistency, but weak evidence strength for scientifically meaningful effects (and vice versa). For example, a set of replications estimating a small average effect size might be statistically consistent with a low-powered original study that estimated a large effect size, yet may provide little evidence that the effects of interest are of scientifically meaningful size. In this case, $P_{\text {orig }}$ would be fairly large, indicating good consistency, but $\widehat{P}_{>q}$ would be small, indicating a low proportion of scientifically meaningful effect sizes. Conversely, a set of replications estimating a moderate effect size may appear statistically inconsistent with an original study estimating a large effect size, but may nevertheless provide strong evidence for scientifically meaningful effect sizes.

The proposed analyses have limitations. We have assumed that the replications yield unbiased estimates, which is often reasonable when the replications are preregistered and conducted by third-party investigators. In contrast, other forms of replications, such as multiple experiments reported in a published, non-registered paper may be subject to the 
same biases seen in published original research (Francis, 2012). As discussed, the metric $P_{\text {orig }}$ assumes that the true effects are normally distributed; this assumption is already often used in pooled effect estimation and is often testable in practice, and simulation results indicated that $P_{\text {orig }}$ in fact rather robust to non-normal distributions. The metric $P_{\text {orig }}$ also relies on accurate statistical estimation of both the pooled effect size and its variance. When estimating these parameters via random-effects meta-analysis, there are many possible choices of heterogeneity estimator, and it is important to choose one that is known to perform well for the effect measure of choice, particularly when the number of replication studies is relatively small (Veroniki et al., 2016). $P_{\text {orig }}$ should be interpreted cautiously if both: (1) the amount of replication data is limited and (2) heterogeneity appears high, as these situations can lead to poor estimation of $\widehat{\tau}^{2}$. We have also suggested diagnostic plots to help identify problems in estimating $\widehat{\tau}^{2}$ that could propagate to $P_{\text {orig }}$ (Section 5). Additionally, we do not recommend using $P_{\text {orig }}$ to conduct a dichotomous "hypothesis test" of consistency (by assessing whether $\left.P_{\text {orig }}<0.05\right)$ between the original study and the replications; rather, $P_{\text {orig }}$ is a continuous measure and is more informative when reported as such.

Simulation results indicated that confidence interval coverage for $\widehat{P}_{>q}$ and the related proportion metrics remained near the nominal $95 \%$ in all scenarios, though point estimates may be biased when there are few replications or small sample sizes in the replications. Therefore, we recommend reporting confidence intervals along with the proportion metrics and using simple bootstrapping-based diagnostics (Section 5) to identify potential bias. When there is little replication data, estimating the amount of heterogeneity can be inherently imprecise (Veroniki et al., 2016). This uncertainty propagates to the confidence intervals for the proportion metrics and, when there is little replication data, may result in confidence intervals that span most or all of the possible range $[0,1]$. Reporting confidence intervals in these settings may nevertheless be informative: a very wide confidence interval may instill appropriate circumspection about what can be learned from the replications, even if $\widehat{\mu}$ itself may have a narrow confidence interval. Additionally, the bootstrapped confidence interval 
may sometimes fail to converge when there are few replication studies or if the threshold $q$ is very far from $\widehat{\mu}$; in these cases, one can try choosing a less extreme threshold.

In summary, the newly proposed metrics assess consistency of the original and replication studies and also assess evidence for effects of scientifically meaningful size while accounting for heterogeneity across the true effects. Such heterogeneity is fairly common in practice and can arise due to differing subject demographics or protocol variations. If reported in many-to-one replication projects, the proposed metrics could help directly and intuitively address the central objectives of replication research. These metrics are mathematically simple but are nevertheless, we believe, a useful supplement to current reporting practices to help quantitatively ground speculation about "replication success". 
REPRODUCIBILITY

All data, materials, and code required to reproduce the applied analyses and simulation study are publicly available, along with all code for the $\mathrm{R}$ packages MetaUtility and Replicate (https://osf.io/ufjg4/). The simulation data can also be browsed interactively (https://mmathur.shinyapps.io/RRR_sims/).

MM conceptualized the research, conducted data analysis and simulations, wrote the $\mathrm{R}$ packages, and led writing. TVW provided key input into the development of these methods and into writing. Both authors approved the final manuscript and declare that they have no conflicts of interest with respect to the authorship or publication of this article.

Cl

MM was supported by National Defense Science and Engineering Graduate Fellowship 32 CFR 168a. TVW was supported by NIH grants ES017876 and CA222147. The funders had no role in the design, conduct, or reporting of this research. 


\section{REFERENCES}

Alogna, V., Attaya, M. K., Aucoin, P., Bahník, Š., Birch, S., Birt, A. R., ... others (2014). Registered replication report: Schooler and Engstler-Schooler (1990). Perspectives on Psychological Science, 9(5), 556-578.

Anderson, S. F., \& Maxwell, S. E. (2016). There's more than one way to conduct a replication study: Beyond statistical significance. Psychological Methods, 21(1), 1.

Andrews, I., \& Kasy, M. (2017). Identification of and correction for publication bias (Tech. Rep.). National Bureau of Economic Research.

Association for Psychological Science. (2018). Ongoing replication projects. https://www .psychologicalscience.org/publications/replication/ongoing-projects. (Accessed: 2018-04-18)

Baumeister, R. F., \& Vohs, K. D. (2016). Misguided effort with elusive implications. Perspectives on Psychological Science, 11(4), 574-575.

Bouwmeester, S., Verkoeijen, P. P., Aczel, B., Barbosa, F., Bègue, L., Brañas-Garza, P., ... others (2017). Registered Replication Report: Rand, Greene, and Nowak (2012). Perspectives on Psychological Science, 12(3), 527-542.

Camerer, C. F., Dreber, A., Forsell, E., Ho, T.-H., Huber, J., Johannesson, M., ... others (2016). Evaluating replicability of laboratory experiments in economics. Science, 351(6280), 1433-1436.

Carpenter, J., \& Bithell, J. (2000). Bootstrap confidence intervals: when, which, what? a practical guide for medical statisticians. Statistics in Medicine, 19(9), 1141-1164.

Cheung, I., Campbell, L., LeBel, E. P., Ackerman, R., Aykutoğlu, B., Bahník, Š., ... others (2016). Registered replication report: Study 1 from Finkel, Rusbult, Kumashiro, \& Hannon (2002). Perspectives on Psychological Science, 11(5), 750-764. 
Cohen, J. (1977). Statistical power analysis for the behavioral sciences. New York: Academic Press.

Cova, F., et al. (2018). Estimating the reproducibility of experimental philosophy. https:// osf.io/7f5hs/. (Accessed: 2018-04-18)

Crandall, C. S., \& Sherman, J. W. (2016). On the scientific superiority of conceptual replications for scientific progress. Journal of Experimental Social Psychology, 66, 93-99.

Davison, A. C., \& Hinkley, D. V. (1997). Bootstrap methods and their application (Vol. 1). Cambridge University Press.

Ebersole, C. R., Atherton, O. E., Belanger, A. L., Skulborstad, H. M., Allen, J. M., Banks, J. B., ... others (2016). Many Labs 3: Evaluating participant pool quality across the academic semester via replication. Journal of Experimental Social Psychology, 67, 68-82.

Ebersole, C.R., et al. (2018). Many Labs 5: Can conducting formal peer review in advance improve reproducibility? https://osf.io/7a6rd/. (Accessed: 2018-04-18)

Eerland, A., Sherrill, A. M., Magliano, J. P., Zwaan, R. A., Arnal, J., Aucoin, P., ... others (2016). Registered replication report: Hart \& Albarracín (2011). Perspectives on Psychological Science, 11(1), 158-171.

Efron, B. (1987). Better bootstrap confidence intervals. Journal of the American Statistical Association, 82(397), 171-185.

Etz, A., \& Vandekerckhove, J. (2016). A Bayesian perspective on the Reproducibility Project: Psychology. PLoS One, 11(2), e0149794.

Fisher, Z., \& Tipton, E. (2015). Robumeta: An R-package for robust variance estimation in meta-analysis. arXiv preprint arXiv:1503.02220.

Gadbury, G. L., \& Iyer, H. K. (2000). Unit-treatment interaction and its practical consequences. Biometrics, $56(3)$, 882-885. 
Gadbury, G. L., Iyer, H. K., \& Allison, D. B. (2001). Evaluating subject-treatment interaction when comparing two treatments. Journal of Biopharmaceutical Statistics, 11 (4), 313-333.

Hagger, M. S., Chatzisarantis, N. L., Alberts, H., Anggono, C. O., Batailler, C., Birt, A. R., ... others (2016). A multilab preregistered replication of the ego-depletion effect. Perspectives on Psychological Science, 11(4), 546-573.

Hardy, R. J., \& Thompson, S. G. (1998). Detecting and describing heterogeneity in metaanalysis. Statistics in Medicine, 17(8), 841-856.

Heckman, J. J., Smith, J., \& Clements, N. (1997). Making the most out of programme evaluations and social experiments: Accounting for heterogeneity in programme impacts. The Review of Economic Studies, 64(4), 487-535.

Hedges, L. V., \& Olkin, I. (1980). Vote-counting methods in research synthesis. Psychological Bulletin, 88(2), 359 .

Hedges, L. V., Tipton, E., \& Johnson, M. C. (2010). Robust variance estimation in meta-regression with dependent effect size estimates. Research Synthesis Methods, 1(1), 39-65.

Higgins, J. P., Thompson, S. G., \& Spiegelhalter, D. J. (2009). A re-evaluation of randomeffects meta-analysis. Journal of the Royal Statistical Society: Series A (Statistics in Society), 172(1), 137-159.

Kenny, D., \& Judd, C. (2018). The unappreciated heterogeneity of effect sizes: implications for power, planning of research, and replication. https://osf.io/s9gfm/. (Accessed: 2018-04-18)

Klein, R. A., Ratliff, K. A., Vianello, M., Adams Jr, R. B., Bahník, Š., Bernstein, M. J., ... others (2014). Investigating variation in replicability. Social Psychology. 
Klein, R.A., et al. (2018). Many Labs 2: investigating variation in replicability across sample and setting. https://osf.io/8cd4r/. (Accessed: 2018-04-18)

Lakens, D. (2013). Calculating and reporting effect sizes to facilitate cumulative science: a practical primer for t-tests and ANOVAs. Frontiers in Psychology, 4.

Lakens, D., Scheel, A. M., \& Isager, P. M. (2018). Equivalence testing for psychological research: A tutorial. Advances in Methods and Practices in Psychological Science, 1(2), 259-269.

Longford, N. T. (1999). Selection bias and treatment heterogeneity in clinical trials. Statistics in Medicine, 18(12), 1467-1474.

Louis, T. A. (1984). Estimating a population of parameter values using bayes and empirical bayes methods. Journal of the American Statistical Association, 79 (386), 393-398.

Lynch, J. G., Bradlow, E. T., Huber, J. C., \& Lehmann, D. R. (2015). Reflections on the Replication Corner: In praise of conceptual replications. International Journal of Research in Marketing, 32(4), 333-342.

Mathur, M. B., \& VanderWeele, T. J. (2019). New metrics for meta-analyses of heterogeneous effects. Statistics in Medicine, 38(8), 1336-1342.

Mathur, M. B., \& VanderWeele, T. J. (in press). Robust metrics and sensitivity analyses for meta-analyses of heterogeneous effects. Epidemiology.

Monin, B. (2016). Be careful what you wish for: Commentary on Ebersole et al. (2016). Journal of Experimental Social Psychology, 67, 95-96.

Monin, B., \& Miller, D. T. (2001). Moral credentials and the expression of prejudice. Journal of Personality and Social Psychology, $81(1), 33$.

Monin, B., Oppenheimer, D. M., Ferguson, M. J., Carter, T. J., Hassin, R. R., Crisp, R. J., .. others (2014). Commentaries and rejoinder on Klein et al.(2014). Social Psychology. 
Nosek, B. A., Spies, J. R., \& Motyl, M. (2012). Scientific utopia: II. Restructuring incentives and practices to promote truth over publishability. Perspectives on Psychological Science, $7(6), 615-631$.

Open Science Collaboration. (2015). Estimating the reproducibility of psychological science. Science, $349(6251)$, aac4716.

Patil, P., Peng, R. D., \& Leek, J. T. (2016). What should researchers expect when they replicate studies? A statistical view of replicability in psychological science. Perspectives on Psychological Science, 11(4), 539-544.

Paule, R. C., \& Mandel, J. (1982). Consensus values and weighting factors. Journal of Research of the National Bureau of Standards, 87(5), 377-385.

Pettigrew, T. F., \& Tropp, L. R. (2008). How does intergroup contact reduce prejudice? meta-analytic tests of three mediators. European Journal of Social Psychology, 38(6), $922-934$.

Raudenbush, S. W., \& Bryk, A. S. (1985). Empirical bayes meta-analysis. Journal of Educational Statistics, 10(2), 75-98.

Rice, K., Higgins, J., \& Lumley, T. (2018). A re-evaluation of fixed effect(s) meta-analysis. Journal of the Royal Statistical Society: Series A (Statistics in Society), 181(1), 205-227.

Riley, R. D., Higgins, J. P., \& Deeks, J. J. (2011). Interpretation of random effects metaanalyses. BMJ, 342, d549.

Rubin, D. B. (1981). Estimation in parallel randomized experiments. Journal of Educational Statistics, 6(4), 377-401.

Schweinsberg, M., Madan, N., Vianello, M., Sommer, S. A., Jordan, J., Tierney, W., ... others (2016). The pipeline project: Pre-publication independent replications of a single laboratory's research pipeline. Journal of Experimental Social Psychology, 66 , 55-67. 
Schweinsberg, M., \& Uhlmann, E. (2018). The Pipeline Project 2. https://osf.io/skq2b/. (Accessed: 2018-04-18)

Shapiro, S., \& Francia, R. (1972). An approximate analysis of variance test for normality. Journal of the American Statistical Association, 67(337), 215-216.

Shapiro, S., \& Wilk, M. B. (1965). An analysis of variance test for normality (complete samples). Biometrika, 52(3/4), 591-611.

Simons, D. J. (2014). The value of direct replication. Perspectives on Psychological Science, $9(1), 76-80$.

Simons, D. J., Holcombe, A. O., \& Spellman, B. A. (2014). An introduction to Registered Replication Reports at Perspectives on Psychological Science. Perspectives on Psychological Science, 9(5), 552-555.

Simonsohn, U. (2015). Small telescopes: Detectability and the evaluation of replication results. Psychological Science, 26(5), 559-569.

Stewart, G. B., Altman, D. G., Askie, L. M., Duley, L., Simmonds, M. C., \& Stewart, L. A. (2012). Statistical analysis of individual participant data meta-analyses: a comparison of methods and recommendations for practice. PloS One, 7(10), e46042.

Sutton, A. J., Abrams, K. R., Jones, D. R., Jones, D. R., Sheldon, T. A., \& Song, F. (2000). Methods for meta-analysis in medical research.

Verhagen, J., \& Wagenmakers, E.-J. (2014). Bayesian tests to quantify the result of a replication attempt. Journal of Experimental Psychology: General, 143(4), 1457.

Veroniki, A., Jackson, D., Viechtbauer, W., Bender, R., Bowden, J., Knapp, G., .. Salanti, G. (2016). Methods to estimate the between-study variance and its uncertainty in meta-analysis. Research Synthesis Methods, 7(1), 55-79. 
Viechtbauer, W. (2010). Conducting meta-analyses in $\mathrm{r}$ with the metafor package. Journal of Statistical Software, 36(3).

Wagenmakers, E.-J., Beek, T., Dijkhoff, L., Gronau, Q. F., Acosta, A., Adams Jr, R., ... others (2016). Registered Replication Report: Strack, Martin, \& Stepper (1988). Perspectives on Psychological Science, $11(6)$, 917-928.

Wang, C.-C., \& Lee, W.-C. (2019). A simple method to estimate prediction intervals and predictive distributions: Summarizing meta-analyses beyond means and confidence intervals. Research Synthesis Methods, 10(2), 255-266. 


\title{
Supplement: New Statistical Metrics for Multisite Replication Projects
}

\author{
Contents
}

\begin{tabular}{|lll}
\hline 1 & Theory and statistical details & 2
\end{tabular}

1.1 Agreement in "statistical significance" . . . . . . . . . . . . . . . . . . . . . . 2

1.2 Estimating the true effect distribution $\ldots \ldots \ldots$. . . . . . . . . . 4

1.3 Derivation of $P_{\text {orig }} \ldots \ldots \ldots \ldots \ldots \ldots$

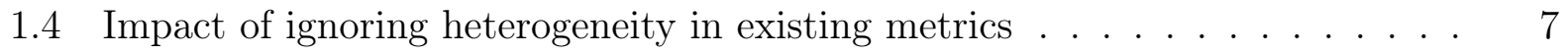

\begin{tabular}{|lll}
2 & Methods for choosing an effect size threshold & 9
\end{tabular}

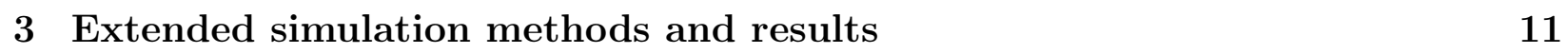

3.1 Methods . . . . . . . . . . . . . . . . . . . . . . 11

3.2 Type I error and power of $P_{\text {orig }} \ldots \ldots \ldots \ldots$

3.3 Bias and root mean square error of $\widehat{P}_{>q} \ldots \ldots \ldots \ldots$

\begin{tabular}{llr}
\hline Software & 18
\end{tabular} 


\section{ThEORY AND STATISTICAL DETAILS}

\subsection{Agreement in "statistical significance"}

Suppose the original study tested the null hypothesis $H_{0}: \mu=\mu_{N}$, where $\mu$ is an unknown population parameter. Consider for now a single replication study, and let $\widehat{\theta}_{\text {orig }}$ and $\widehat{\theta}_{\text {rep }}$ be estimates of $\mu$ from the original and replication study, respectively. Assume that under both the null and alternative hypotheses, $\widehat{\theta}_{\text {orig }}$ and $\widehat{\theta}_{\text {rep }}$ are approximately and independently normal with a common mean but potentially different standard errors:

$$
\begin{aligned}
\widehat{\theta}_{\text {orig }} \sim N\left(\mu, S E_{\text {orig }}^{2}\right) \amalg \widehat{\theta}_{\text {rep }} \sim N\left(\mu, S E_{\text {rep }}^{2}\right) \\
\Rightarrow \widehat{\theta}_{\text {rep }}-\widehat{\theta}_{\text {orig }} \sim N\left(0, S E_{\text {rep }}^{2}+S E_{\text {orig }}^{2}\right)
\end{aligned}
$$

where $\amalg$ denotes statistical independence. (Critically, this setup does not allow for heterogeneity in that it assumes that the replication and original studies measure the same true effect, $\mu$. Section 1.4 demonstrates the impact of this stringent assumption.) Considering first the case in which the original estimate is above the null (i.e., $\widehat{\theta}_{\text {orig }}-\mu_{N}>0$ ), we can derive the probability of a "significant" replication estimate that is also above the null $\left(\widehat{\theta}_{\text {rep }}-\mu_{N}>0\right)$ given the original estimate and its true standard error $\left(\widehat{\theta}_{\text {orig }}\right.$ and $\left.S E_{\text {orig }}\right)$. Let $c_{\alpha}=\Phi^{-1}(1-\alpha / 2)$ be the critical value of the normalized test statistic (e.g., 1.96 for $\alpha=0.05)$. Standardize $\widehat{\theta}_{\text {rep }}$ to construct the usual standard-normal test statistic and express 
the desired probability as:

$$
\begin{aligned}
& P\left(\frac{\widehat{\theta}_{\text {rep }}-\mu_{N}}{S E_{\text {rep }}}>c_{\alpha} \mid \widehat{\theta}_{\text {orig }}, S E_{\text {orig }}\right)=P\left(\widehat{\theta}_{\text {rep }}>c_{\alpha} S E_{\text {rep }}+\mu_{N} \mid \widehat{\theta}_{\text {orig }}, S E_{\text {orig }}\right) \\
& =P(\underbrace{\frac{\widehat{\theta}_{\text {rep }}-\widehat{\theta}_{\text {orig }}}{\sqrt{S E_{\text {rep }}^{2}+S E_{\text {orig }}^{2}}}}_{N(0,1)}>\frac{c_{\alpha} S E_{\text {rep }}+\mu_{N}-\widehat{\theta}_{\text {orig }}}{\sqrt{S E_{\text {rep }}^{2}+S E_{\text {orig }}^{2}}}) \\
& =1-\Phi\left(\frac{c_{\alpha} S E_{\mathrm{rep}}+\mu_{N}-\widehat{\theta}_{\mathrm{orig}}}{\sqrt{S E_{\mathrm{rep}}^{2}+S E_{\mathrm{orig}}^{2}}}\right) \\
& \approx 1-\Phi\left(\frac{c_{\alpha} \widehat{S E}_{\mathrm{rep}}+\mu_{N}-\widehat{\theta}_{\mathrm{orig}}}{\sqrt{\widehat{S E}_{\mathrm{rep}}^{2}+\widehat{S E}_{\mathrm{orig}}^{2}}}\right)
\end{aligned}
$$

where the second equality follows from Eq. 1.1 and the final approximation follows by substituting estimated standard errors for their true counterparts. Similarly, considering the case in which the original estimate is below the null $\left(\widehat{\theta}_{\text {orig }}-\mu_{N}<0\right)$, the probability of a "significant" replication estimate that is also below the null is:

$$
\begin{aligned}
& P\left(\frac{\widehat{\theta}_{\text {rep }}-\mu_{N}}{S E_{\text {rep }}}<-c_{\alpha} \mid \widehat{\theta}_{\text {orig }}, S E_{\text {orig }}\right)=P\left(\widehat{\theta}_{\text {rep }}<-c_{\alpha} S E_{\text {rep }}+\mu_{N} \mid \widehat{\theta}_{\text {orig }}, S E_{\text {orig }}\right) \\
& =P(\underbrace{\frac{\widehat{\theta}_{\text {rep }}-\widehat{\theta}_{\text {orig }}}{\sqrt{S E_{\text {rep }}^{2}+S E_{\text {orig }}^{2}}}}_{N(0,1)}<\frac{-c_{\alpha} S E_{\text {rep }}+\mu_{N}-\widehat{\theta}_{\text {orig }}}{\sqrt{S E_{\text {rep }}^{2}+S E_{\text {orig }}^{2}}}) \\
& \approx \Phi\left(\frac{-c_{\alpha} \widehat{S E}_{\mathrm{rep}}+\mu_{N}-\widehat{\theta}_{\mathrm{orig}}}{\sqrt{\widehat{S E}_{\mathrm{rep}}^{2}+\widehat{S E}_{\text {orig }}^{2}}}\right)
\end{aligned}
$$

When there are multiple replications (in either a many-to-one or one-to-one design), one can simply apply either Equation 1.3 or 1.4 to each replication study depending on the sign of the relevant original estimate. 


\subsection{Estimating the true effect distribution}

We assume that the replication studies estimate (with statistical error) potentially different true effect sizes that follow a normal distribution. The distribution of true effects is distinct from the observed distribution of replication estimates; the latter is more variable due to statistical error in the replication studies. The proposed analyses therefore begin by using the replication studies to estimate the mean and variance of the distribution of true effects using one of two straightforward modeling approaches (though these are not exhaustive possibilities). Both approaches begin with shared assumptions. Let $\widehat{\theta}_{\text {rep,i }}$ denote the point estimate in the $i^{t h}$ replication such that $\widehat{\theta}_{\text {rep }, \mathrm{i}}=\mu+\gamma_{i}+\epsilon_{i}$, where $\gamma_{i} \sim N\left(0, \tau^{2}\right)$ denotes deviations of site-specific true effects from the grand mean $(\mu)$ and $\epsilon_{i} \sim N\left(0, S E_{\text {rep,i }}^{2}\right)$ denotes statistical error due to finite sample sizes in the replication studies. Assume that $\gamma_{i}$ and $\epsilon_{i}$ are independent. That is, the true effect in replication site $i$ is $\mu+\gamma_{i}$, which is normal with mean $\mu$ and variance $\tau^{2}$. Its estimate, incorporating additional error due to $\epsilon_{i}$, is $\widehat{\theta}_{\text {rep,i }}$ and is marginally normal with mean $\mu$ and variance $\tau^{2}+S E_{\mathrm{rep}, \mathrm{i}}^{2}$.

To estimate $\mu$ and $\tau^{2}$, one option is compute an point estimate within each site (for example, using the same model as in the original study) and then to conduct a random-effects meta-analysis on these site-level summary measures. Such analyses are already commonplace in many-to-one designs. One can then use the meta-analytic pooled estimate as $\widehat{\mu}$ and the heterogeneity estimate of the variance of the true effects as $\widehat{\tau}^{2}$. A second option, which avoids aggregating data by site prior to analysis, is to fit a mixed model to the observation-level data with independent, identically normal random intercepts and slopes by site; this is a form of individual participant data meta-analysis (Stewart et al., 2012). For example, suppose the original study used ordinary least squares regression to estimate the effect $\left(\beta_{1}\right)$ of a binary experimental manipulation $X$ on a continuous dependent variable $Y$ with the usual specification $Y_{j}=\beta_{0}+\beta_{1} X_{j}+\epsilon_{j}$ for subjects $j=1, \cdots, N_{\text {orig }}$ and with the error terms $\epsilon_{j}$ assumed independent and identically ("iid") normal. Then, for the replications, one possible 
mixed model specification is:

$$
\begin{aligned}
& Y_{i j}=\alpha_{0}+\zeta_{0 i}+\alpha_{1} X_{i j}+\zeta_{1 i} X_{i j}+\epsilon_{i j}^{*} \\
& \zeta_{0 i} \sim_{i i d} N\left(0, \sigma_{\zeta_{0}}^{2}\right) \amalg \zeta_{1 i} \sim_{i i d} N\left(0, \sigma_{\zeta_{1}}^{2}\right) \amalg \epsilon_{i j}^{*} \sim_{i i d} N\left(0, \sigma_{\epsilon^{*}}^{2}\right)
\end{aligned}
$$

where $i$ indexes sites. Then, we can estimate $\mu$ (the average true effect of $X$ across all sites) using the usual maximum likelihood or restricted maximum likelihood estimate, $\widehat{\alpha}_{1}$. We can estimate $\tau^{2}$ (the variance of the true effects of $X$ across all sites) with $\widehat{\sigma}_{\zeta_{1}}^{2}$. Depending on the experimental design, of course, a different mixed model specification may be warranted (for example, with additional random terms by subject) as long as it retains the normal assumption on the effect sizes across sites and yields unbiased, approximately normally distributed, and approximately independent estimates of $\mu$ and $\tau^{2}$. Specifications that do not pre-aggregate data within sites may often be more statistically efficient than the meta-analytic approach, but the meta-analysis method may sometimes provide more flexibility because it models the effect sizes rather than the dependent variable itself. Lastly, a third possible modeling approach could simply ignore site and fit the same analysis model as was used in the original study, but we do not recommend this approach because clustering within sites will likely violate statistical assumptions regarding conditionally independent residuals, such specifications preclude estimation of $\tau^{2}$, and they can lead to bias due to Simpson's Paradox (Rücker \& Schumacher, 2008).

\subsection{Derivation of $P_{\text {orig }}$}

Given the estimates $\widehat{\mu}$ and $\widehat{\tau}^{2}$ from the above development, we can derive the probability that, if the original study and replications come from the same, potentially heterogeneous distribution of true effects, the original study would estimate an effect size as extreme or more extreme than its actual estimate. As above, let $\widehat{\theta}_{\text {orig }}$ be the point estimate in the original study and $S E_{\text {orig }}$ its standard error. Letting $\widehat{\theta}^{*}$ be a random variable denoting the effect estimate 
in an arbitrary study with the same standard error as the original, we first consider the distribution of $\widehat{\theta}^{*}-\widehat{\mu}$. Assume that $\widehat{\mu} \sim N\left(\mu, S E_{\widehat{\mu}}^{2}\right)$; that is, the estimate is approximately unbiased and normal. (This holds for both the meta-analysis and the mixed model approaches above under standard assumptions.) Since $\widehat{\theta}^{*} \sim N\left(\mu, \tau^{2}+S E_{\text {orig }}^{2}\right)$ independently of $\widehat{\mu}$, we can derive the first proposed metric as follows:

$$
\begin{aligned}
& \widehat{\theta}^{*}-\widehat{\mu} \sim N\left(0, \tau^{2}+S E_{\text {orig }}^{2}+S E_{\widehat{\mu}}^{2}\right) \\
& P\left(\left|\widehat{\theta}^{*}-\widehat{\mu}\right| \geq\left|\widehat{\theta}_{\text {orig }}-\widehat{\mu}\right|\right)=P\left(\widehat{\theta}^{*}-\widehat{\mu} \geq\left|\widehat{\theta}_{\text {orig }}-\widehat{\mu}\right|\right)+P\left(\widehat{\theta}^{*}-\widehat{\mu} \leq-\left|\widehat{\theta}_{\text {orig }}-\widehat{\mu}\right|\right)
\end{aligned}
$$

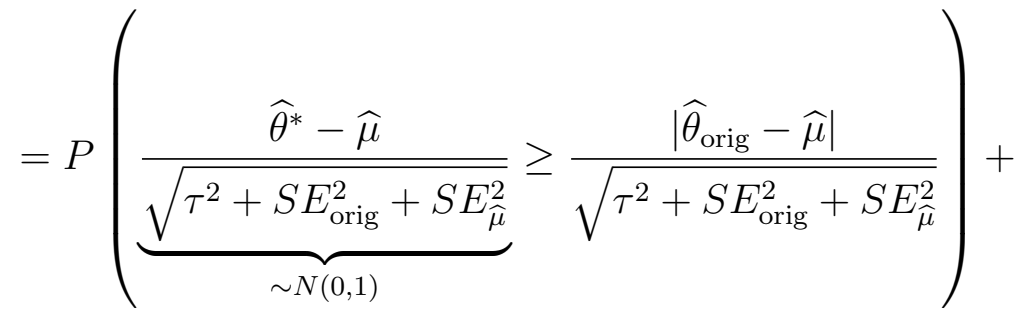

$$
\begin{aligned}
& P(\underbrace{\frac{\widehat{\theta}^{*}-\widehat{\mu}}{\sqrt{\tau^{2}+S E_{\text {orig }}^{2}+S E_{\widehat{\mu}}^{2}}}}_{\sim N(0,1)} \leq \frac{-\left|\widehat{\theta}_{\text {orig }}-\widehat{\mu}\right|}{\sqrt{\tau^{2}+S E^{2}(\widehat{\mu})+S E_{\widehat{\mu}}^{2}}}) \\
& =1-\Phi\left(\frac{\left|\widehat{\theta}_{\text {orig }}-\widehat{\mu}\right|}{\sqrt{\tau^{2}+S E_{\text {orig }}^{2}+S E_{\widehat{\mu}}^{2}}}\right)+\Phi\left(\frac{-\left|\widehat{\theta}_{\text {orig }}-\widehat{\mu}\right|}{\sqrt{\tau^{2}+S E_{\text {orig }}^{2}+S E_{\widehat{\mu}}^{2}}}\right) \\
& =2 \times\left(1-\Phi\left(\frac{\left|\widehat{\theta}_{\text {orig }}-\widehat{\mu}\right|}{\sqrt{\tau^{2}+S E_{\text {orig }}^{2}+S E_{\widehat{\mu}}^{2}}}\right)\right)
\end{aligned}
$$

We arrive at the approximation in the main text (i.e., $P_{\text {orig }}$ ) by substituting the estimates $\widehat{S E}_{\text {orig }}$ and $\widehat{S E}_{\widehat{\mu}}$ for the true parameters.

We now show that $P_{\text {orig }}$ subsumes Patil et al. (2016)'s prediction interval in the sense that if there is a single replication study, if we assume there is no heterogeneity, and if we dichotomize $P_{\text {orig }}$ at $\alpha=0.05$, we mathematically recover the prediction interval. In Equation 1.5. set the left-hand side equal to 0.05 (for a $95 \%$ prediction interval) and $\tau^{2}=0$ (for no heterogeneity). Let $\theta_{0.05}^{*}$ be the hypothetical value for the replication point estimate that 
marks the lower or upper boundary of the $95 \%$ prediction interval. Since the prediction interval concerns a single replication study, set $\widehat{\mu}=\theta_{0.05}^{*}$ and $\widehat{S E}_{\widehat{\mu}}^{2}=\widehat{S E}_{\text {rep }}^{2}$. Thus, we can solve for the boundary values of the prediction interval, i.e., the pair of hypothetical replication estimates that would be sufficiently extreme to make the probability on the left-hand side equal to 0.05 :

$$
\begin{aligned}
0.05 & =2 \times\left(1-\Phi\left(\frac{\left|\widehat{\theta}_{\text {orig }}-\theta_{0.05}^{*}\right|}{{\sqrt{\widehat{S E}_{\text {orig }}^{2}+\widehat{S E}_{\text {rep }}^{2}}}^{2}}\right)\right) \\
\left|\widehat{\theta}_{\text {orig }}-\theta_{0.05}^{*}\right| & =\Phi^{-1}(0.975) \sqrt{\widehat{S E}_{\text {orig }}^{2}+\widehat{S E}_{\text {rep }}^{2}} \\
\widehat{\theta}_{\text {orig }}-\theta_{0.05}^{*} & = \pm \Phi^{-1}(0.975) \sqrt{\widehat{S E}_{\text {orig }}^{2}+\widehat{S E}_{\text {rep }}^{2}} \\
\theta_{0.05}^{*} & =\widehat{\theta}_{\text {orig }} \pm \Phi^{-1}(0.975){\sqrt{\widehat{S E}_{\text {orig }}^{2}+\widehat{S E}_{\text {rep }}^{2}}}
\end{aligned}
$$

which is exactly Patil et al. (2016)'s prediction interval.

\subsection{Impact of ignoring heterogeneity in existing metrics}

We now show that ignoring heterogeneity when estimating the expected "significance agreement" can underestimate or overestimate consistency when there truly is heterogeneity. We begin by generalizing Equation 1.1 (which ignores heterogeneity) to accommodate heterogeneity via the same framework developed in Section 1.2 , assuming normally distributed true effects:

$$
\begin{aligned}
& \widehat{\theta}_{\text {orig }} \sim N\left(\mu, \tau^{2}+S E_{\text {orig }}^{2}\right) \amalg \widehat{\theta}_{\text {rep }} \sim N\left(\mu, \tau^{2}+S E_{\text {rep }}^{2}\right) \\
& \Rightarrow \widehat{\theta}_{\text {rep }}-\widehat{\theta}_{\text {orig }} \sim N\left(0,2 \tau^{2}+S E_{\text {rep }}^{2}+S E_{\text {orig }}^{2}\right)
\end{aligned}
$$

For an original estimate above the null, we can compute the probability of "significance 
agreement" allowing for heterogeneity as:

$$
\begin{aligned}
& P\left(\frac{\widehat{\theta}_{\text {rep }}-\mu_{N}}{S E_{\text {rep }}}>c_{\alpha} \mid \widehat{\theta}_{\text {orig }}, S E_{\text {orig }}\right)=P\left(\widehat{\theta}_{\text {rep }}>c_{\alpha} S E_{\text {rep }}+\mu_{N} \mid \widehat{\theta}_{\text {orig }}, S E_{\text {orig }}\right) \\
& =P(\underbrace{\frac{\widehat{\theta}_{\text {rep }}-\widehat{\theta}_{\text {orig }}}{\sqrt{2 \tau^{2}+S E_{\text {rep }}^{2}+S E_{\text {orig }}^{2}}}}_{N(0,1)}>\frac{c_{\alpha} \widehat{S E}_{\text {rep }}+\mu_{N}-\widehat{\theta}_{\text {orig }}}{\sqrt{2 \tau^{2}+S E_{\text {rep }}^{2}+S E_{\text {orig }}^{2}}}) \\
& \approx 1-\Phi\left(\frac{c_{\alpha} \widehat{S E}_{\text {rep }}+\mu_{N}-\widehat{\theta}_{\text {orig }}}{\sqrt{2 \tau^{2}+\widehat{S E}_{\text {rep }}^{2}+\widehat{S E}_{\text {orig }}^{2}}}\right)
\end{aligned}
$$

The only difference between this expression and Equation 1.2 (which had assumed no heterogeneity) is the $2 \tau^{2}$ term in the denominator. Since the presence of heterogeneity implies that $2 \tau^{2}>0$, this probability exceeds that in Equation 1.2 when $c_{\alpha} \widehat{S E}_{\text {rep }}+\mu_{N}-\widehat{\theta}_{\text {orig }}>0$ and otherwise is less than that in Equation 1.2 . Thus, when $c_{\alpha} \widehat{S E}_{\text {rep }}+\mu_{N}-\widehat{\theta}_{\text {orig }}>0$, analyses that ignore heterogeneity will underestimate consistency between the replication and the original study, and otherwise, they will overestimate consistency. The case in which the original estimate is below the null is symmetrical, so is omitted.

When there is heterogeneity, the prediction interval will be too narrow. Using the previous result showing equivalence of $P_{\text {orig }}$ with the prediction interval when there is no heterogeneity, we can set $\tau^{2}=0$ in Equation 1.5 to yield the $p$-value counterpart to the prediction interval. Since Equation 1.5 is strictly increasing in $\tau^{2}$, constraining $\tau^{2}=0$ in this expression yields a lower $p$-value than allowing $\tau^{2}>0$. Thus, if there is heterogeneity, the $p$-value counterpart to the prediction interval is an underestimate. By the duality of $p$-values and intervals, the prediction interval is therefore too narrow when $\tau^{2}>0$. 


\section{Methods For ChOOSING AN EFFECT Size THRESHOLD}

Much existing work, spanning a variety of disciplinary perspectives, has discussed how to choose thresholds for scientifically meaningful effect sizes. Crosby et al. (2003) provide an excellent review and examples of numerous methods in the context of health outcomes. In particular, they discuss a variety of "anchoring-based" methods in which an effect size threshold is chosen by relating the outcome measure to external benchmarks bearing immediate scientific or policy relevance. Within psychology, this approach may be particularly relevant for applied or intervention studies; for example, when investigating effects of educational interventions, a minimum effect size threshold could be determined in relation to differences in the outcome (academic achievement) between naturally-occurring subject groups (such as children attending low- versus high-performing schools or children of different ages; Hill et al. (2008)). Numerous other types of external "anchoring" criteria have also been used in the health outcomes literature (Crosby et al., 2003).

When the aggregate public impact of an outcome (such as juvenile delinquency) is the primary concern, investigators could draw upon the extensive literature on cost-effectiveness decision rules in selecting an effect size threshold. For example, much existing work has discussed or empirically quantified the cost threshold at which societies or individuals are willing to pay for a specific improvement in physical or mental health, such as an addition of one quality-adjusted life-year (e.g., Braithwaite et al. (2008); Eichler et al. (2004)). Such findings could be used to "convert" hypothetical statistical effect sizes for a given outcome to a concrete financial scale, such as dollars. A minimum effect size threshold could then be defined in relation to the utility, expressed in dollars, of the intervention or exposure of interest.

In contrast, in disciplines such as clinical psychology, the original study may investigate an effect in which individuals' subjective experience of distress or pain is the primary concern (instead of, or in addition to, aggregate public impact). In this case, it may be useful to 
set the threshold as the minimum effect size that is subjectively perceptible Jaeschke et al. (1989); Lakens et al. (2018); Norman et al. (2003); Redelmeier et al. (1996)). A systematic review considered 62 studies that attempted to estimate such thresholds for a wide variety of health outcomes, for example by relating patients' subjective self-assessments to objective measurements of health condition severity (Norman et al., 2003). This review found that $d=0.50$ was a surprisingly consistent minimally detectable effect size for health outcomes, perhaps reflecting fundamental mechanisms of human sensory discrimination or constraints on categorical discrimination due to working memory capacity. For ease of comparison to other statistical measures of effect size, the threshold $d=0.50$ is approximately equivalent (under some distributional assumptions) to an odds ratio of 2.5 or to a risk ratio of 1.6 Chinn (2000); VanderWeele (2017)). However, it is important to note that an intervention that has only small effects on the individual level, even ones that are not subjectively perceptible, may still have very substantial impacts on a population level; thus, as described above, much lower thresholds might often be considered.

While the above considerations may work well for applied or interventional psychology, many replication efforts to date have focused on classic experimental psychology, conducted using stylized tasks (such as a Stroop task or Monin \& Miller (2001)'s hypothetical hiring task) in order to examine basic mechanisms of, for example, cognition or perception. Although some of the above considerations are harder to apply in these classic experimental contexts, external benchmarks could still be determined using effect sizes on similar experimental tasks, preferably those estimated by meta-analyses of existing literature. For example, a metaanalysis of the enormous literature on intergroup contact and prejudice estimated a pooled effect size of $r=-0.21$ among all study designs and $r=-0.33$ among experimental studies (Pettigrew \& Tropp, 2008). We might treat experimental intergroup-contact interventions as a "gold standard" representing the effect sizes on prejudice that are achievable through purposefully designed interventions. In contrast, the proposed moral credentialing effect is not a designed intervention on prejudice but rather a specific, potentially more subtle, cognitive 
mechanism of prejudice. Thus, to select an effect size threshold for moral credentialing, we might somewhat reduce the magnitude of the gold-standard interventions to, for example, $|r|=0.20$ or $|r|=0.10$. (Additionally, the latter threshold is often considered a standard benchmark for a "small" effect size (Cohen, 1977).)

\section{EXTENDED SIMULATION METHODS AND RESUlTS}

\subsection{Methods}

Figure S1] shows true effects simulated from each of the four distributions for each value of $\tau^{2}$.

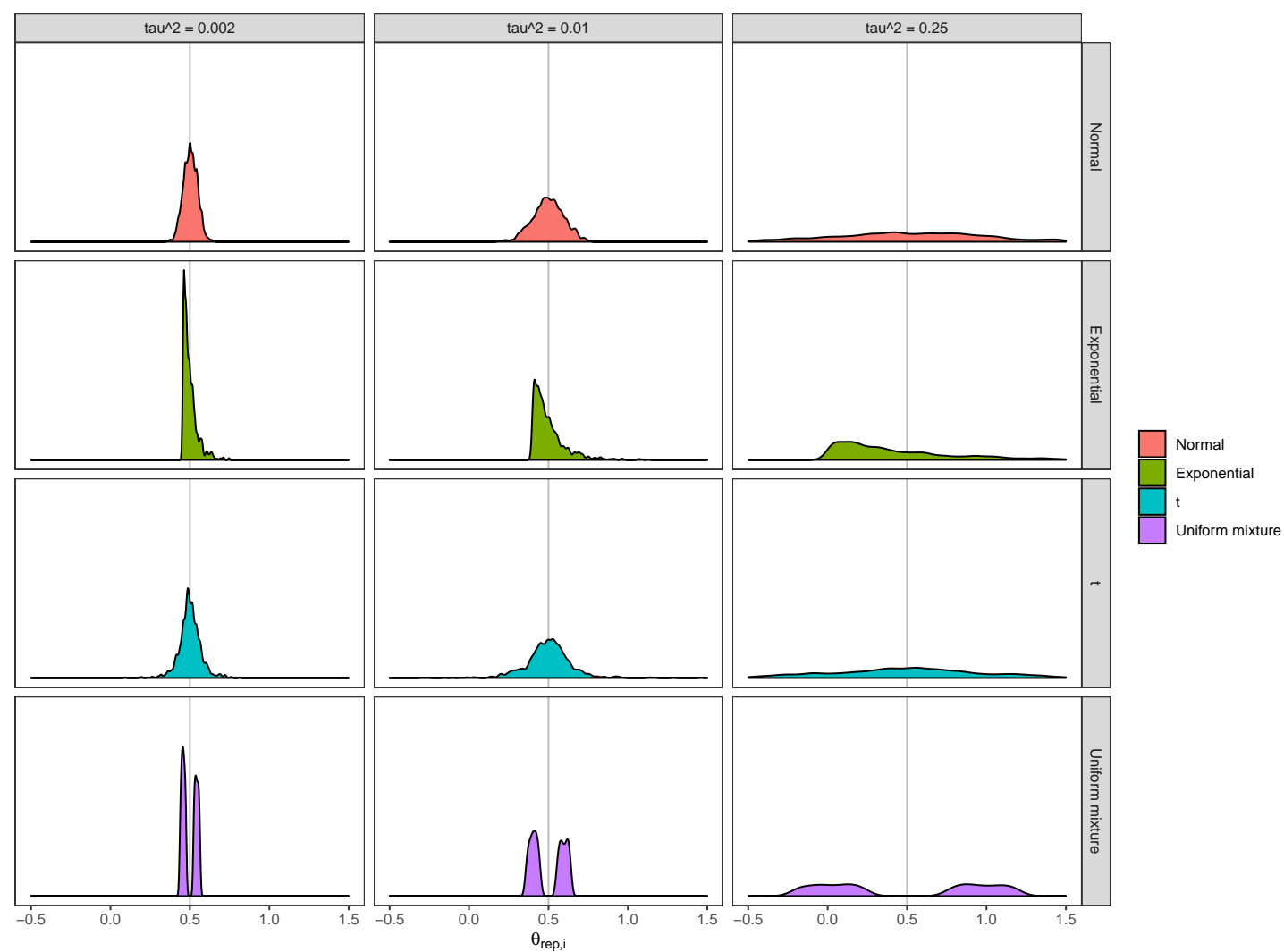

Figure S1: Simulated true effect sizes from the four true effect distributions with varying heterogeneity 


\subsection{Type I error and power of $P_{\text {orig }}$}
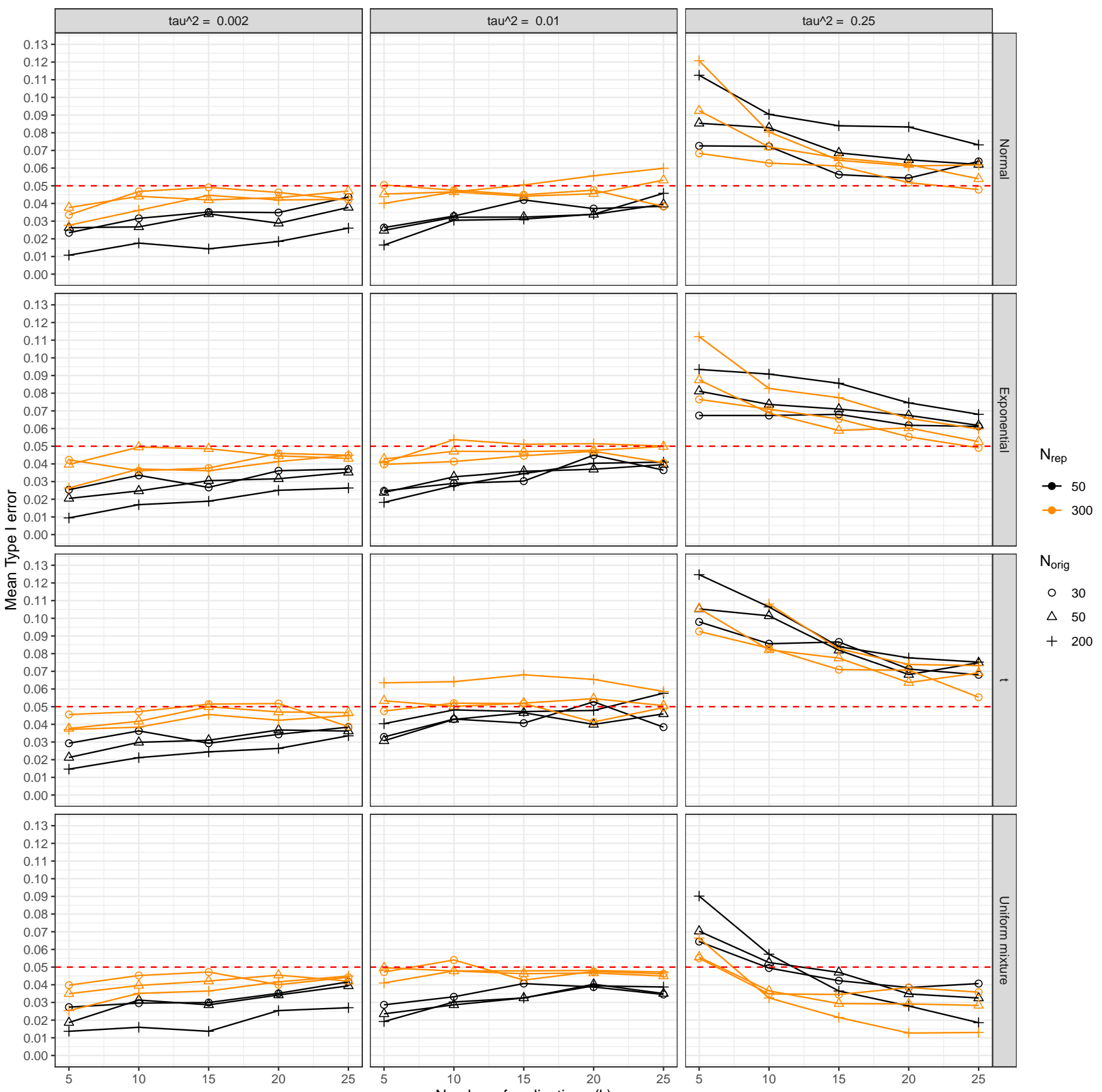

Figure S2: Type I error of $P_{\text {orig }}$ (scenarios with $\Delta=0$ ) 

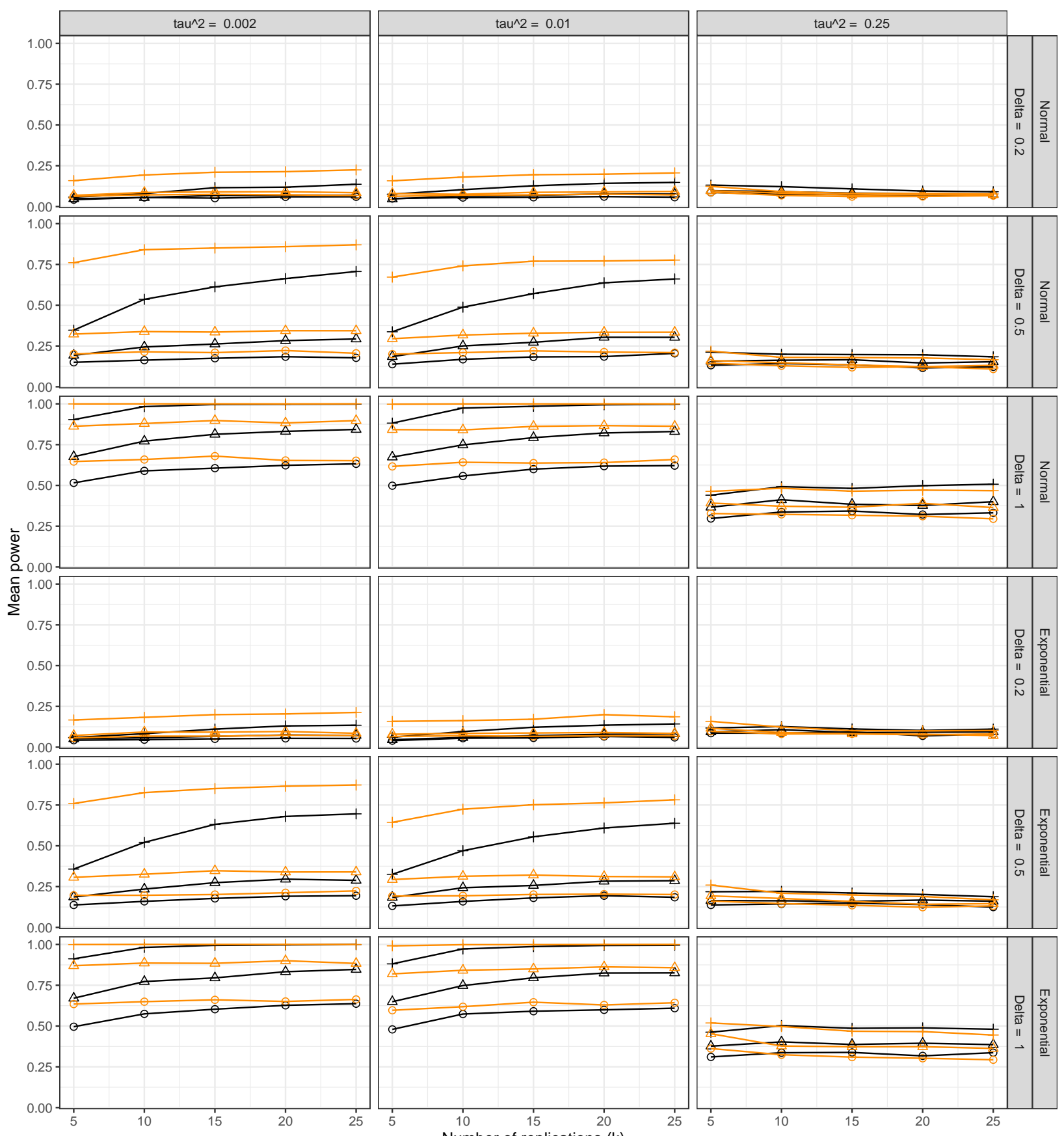

$\mathrm{N}_{\text {rep }}$

$\rightarrow 50$

- 300
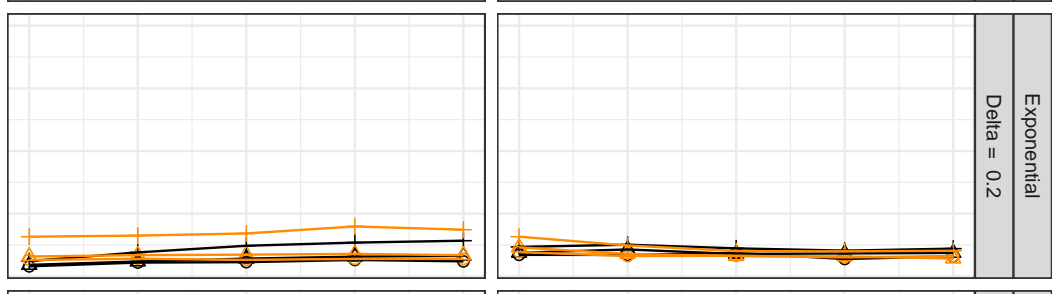

$\mathrm{N}_{\text {orig }}$

$\circ 30$

$\triangle 50$

$+200$
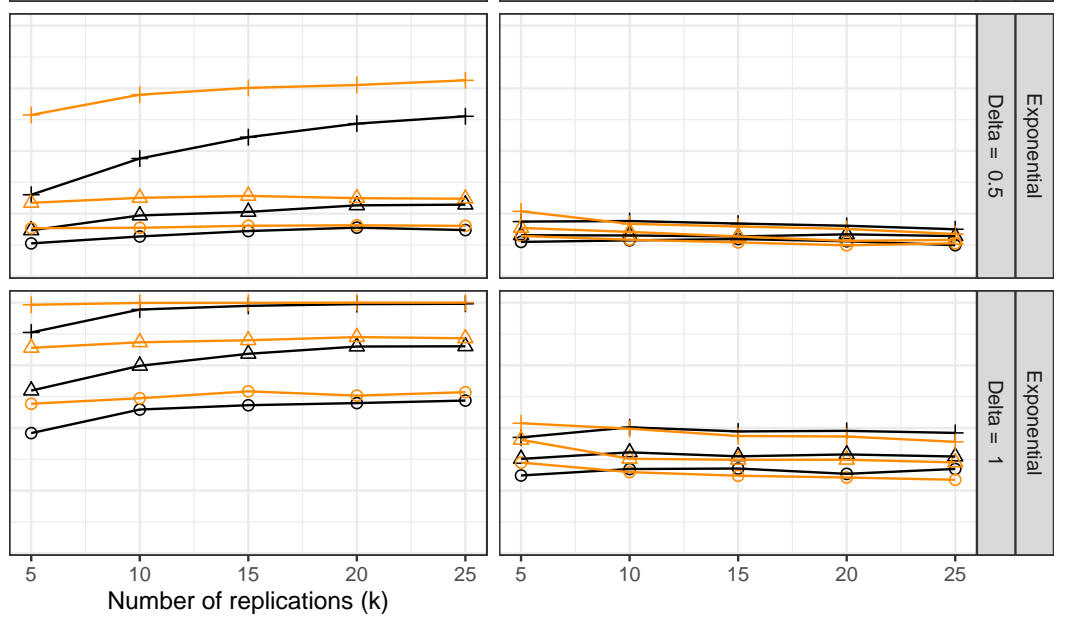

Figure S3: Power of $P_{\text {orig }}$ for normal and exponential distributions (scenarios with $\Delta>0$ ) 

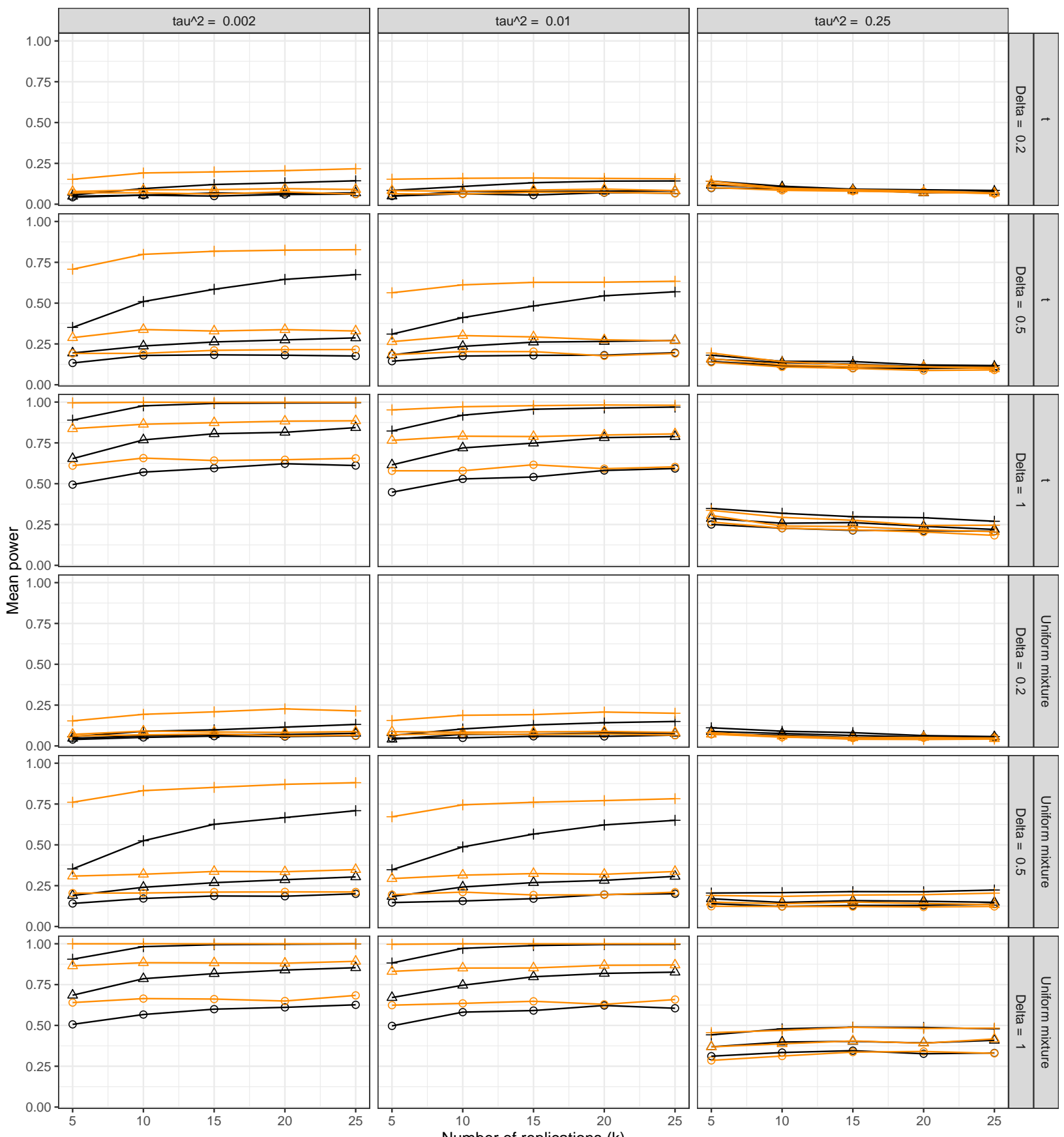

$N_{\text {orig }}$

$\circ 30$

$\triangle 50$

$+200$
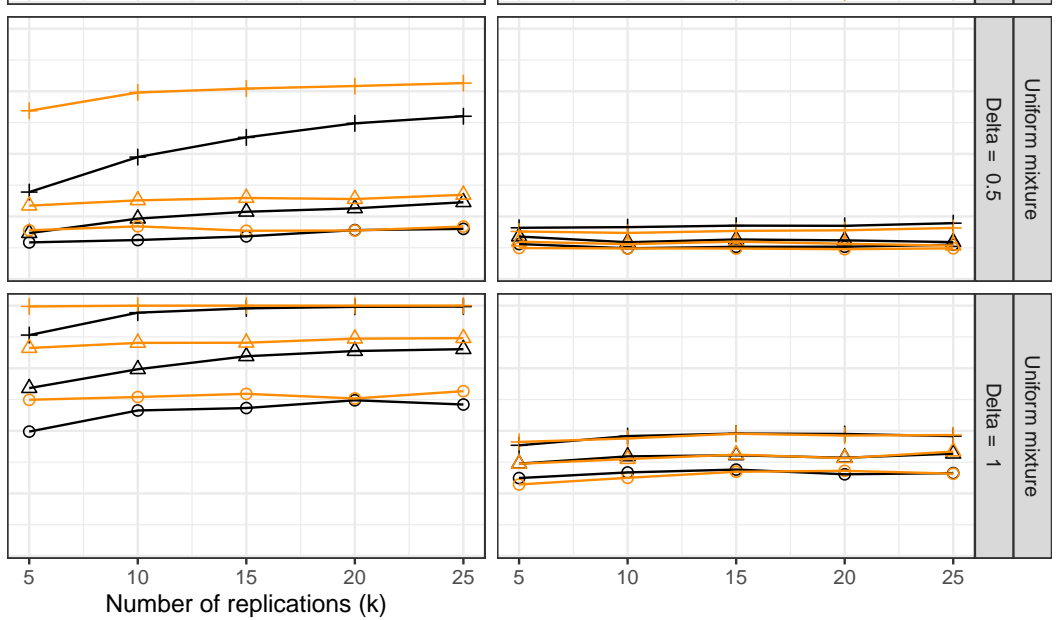

Figure S4: Power of $P_{\text {orig }}$ for $t$ and uniform mixture distributions (scenarios with $\Delta>0$ ) 
3.3. Bias and root mean square error of $\widehat{P}_{>q}$
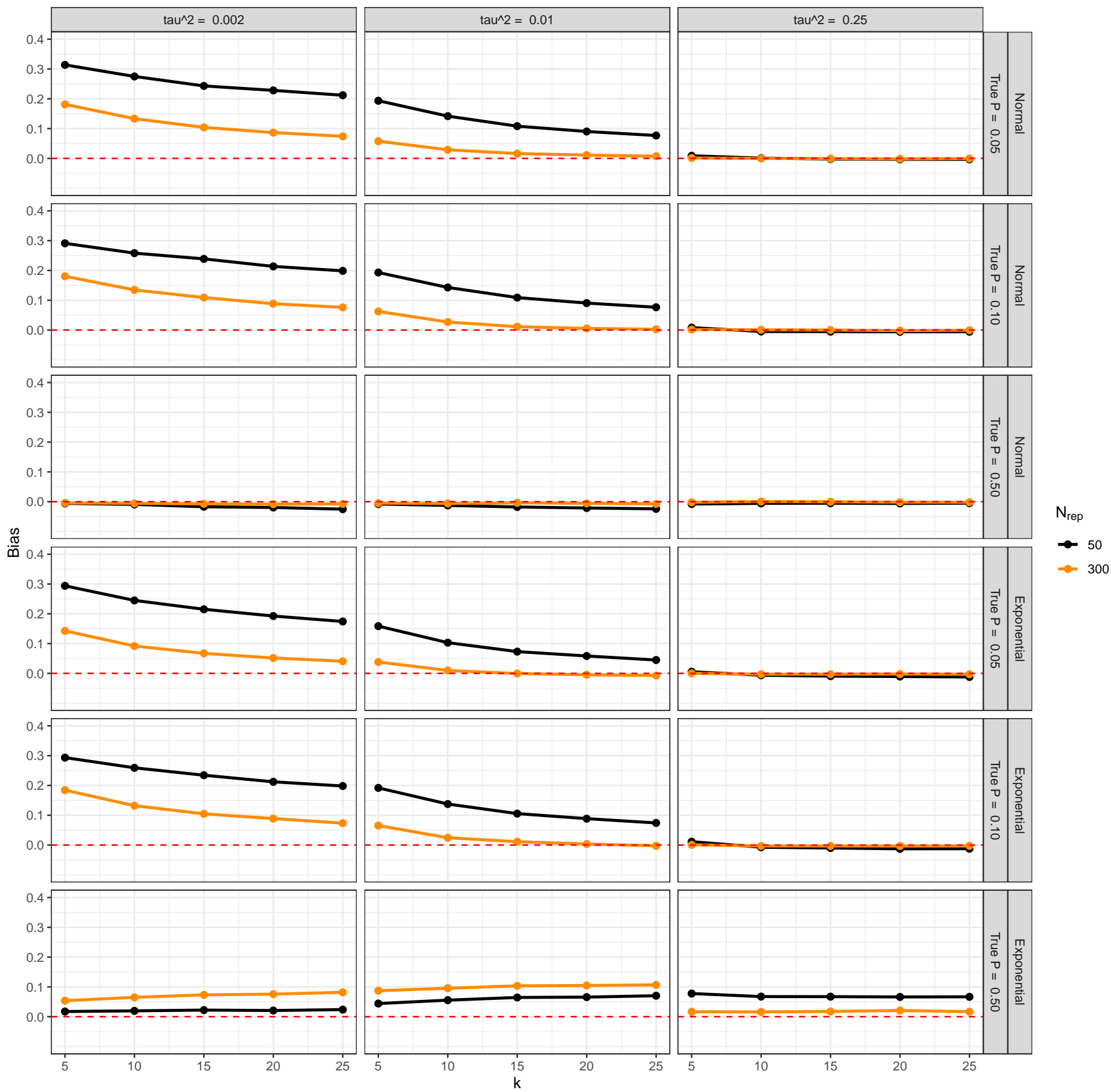

Figure S5: Bias of $\widehat{P}_{>q}$ for normal and exponential distributions 

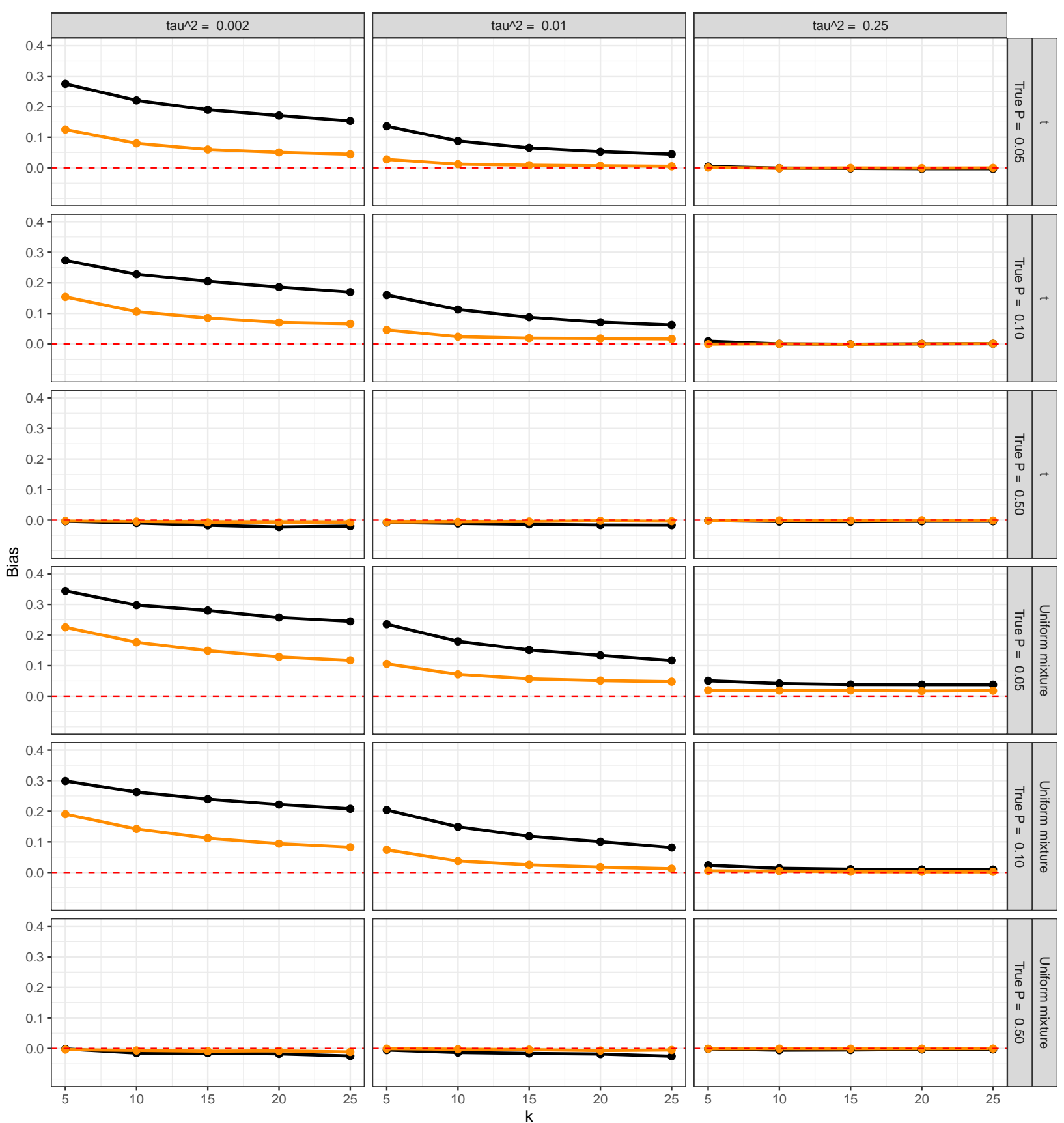

$\mathrm{N}_{\text {rep }}$
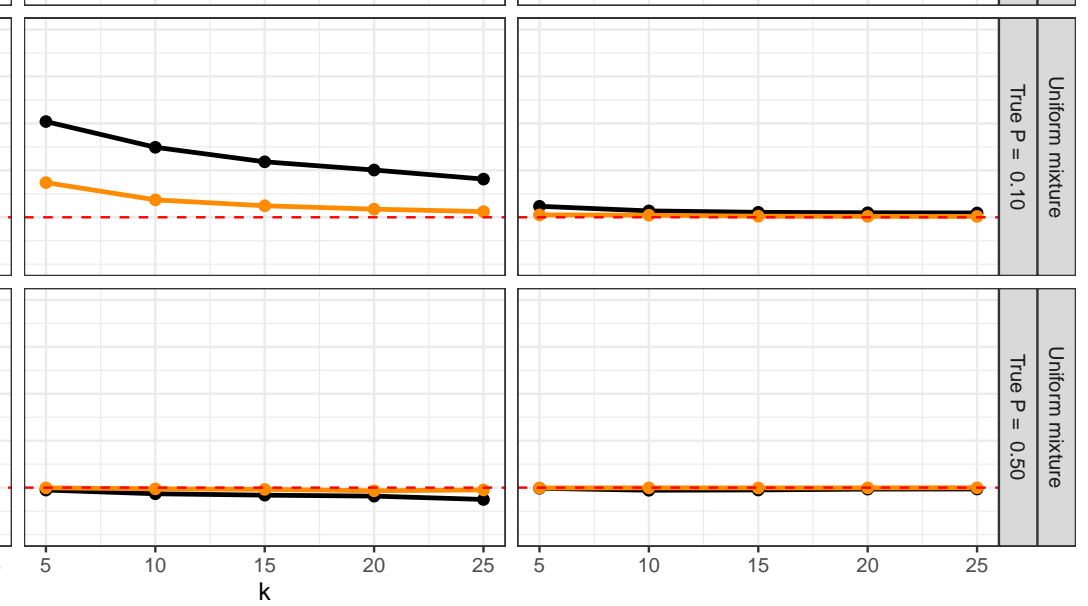

Figure S6: Bias of $\widehat{P}_{>q}$ for $t$ and uniform mixture distributions 

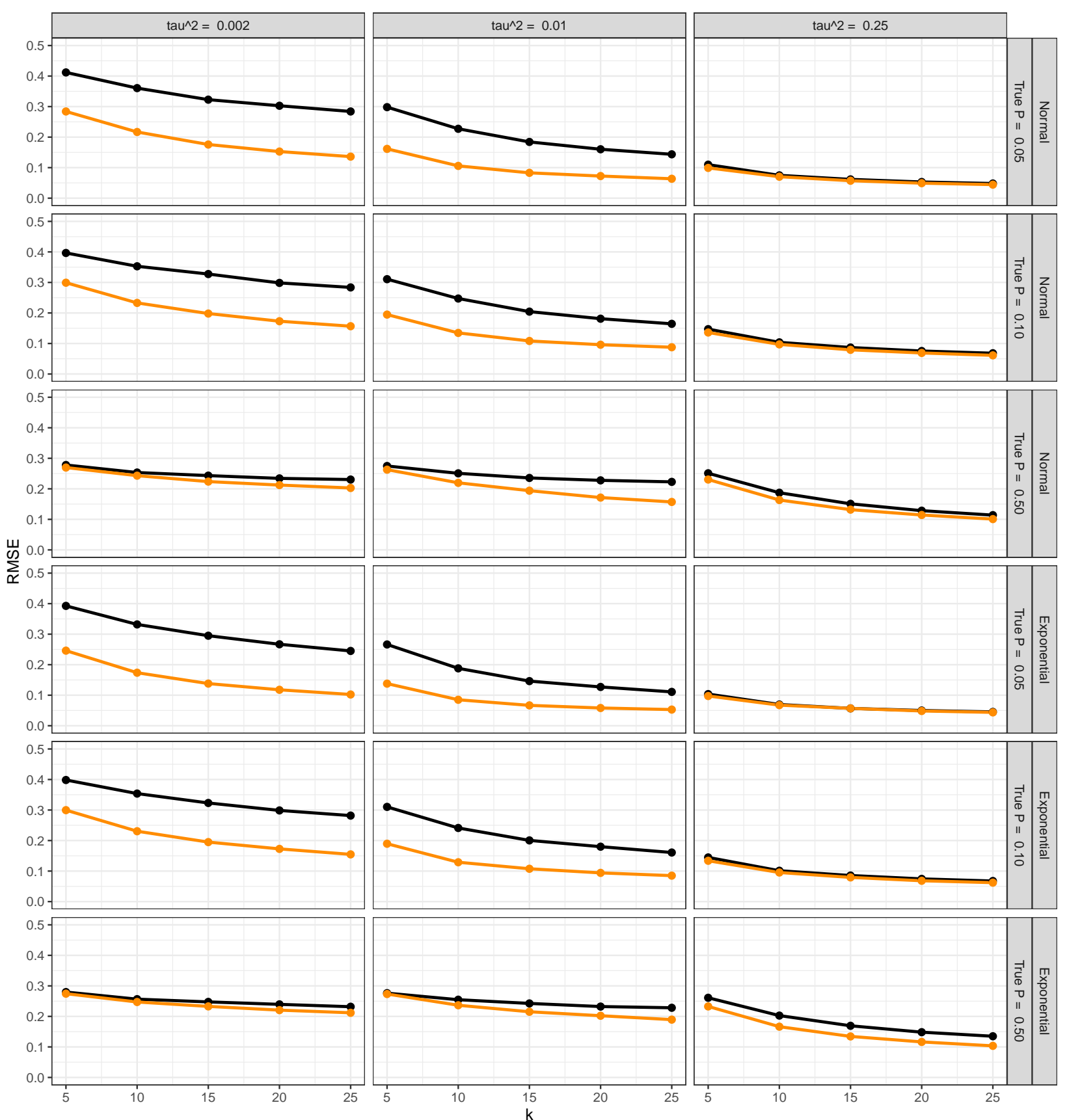

$\mathrm{N}_{\text {rep }}$
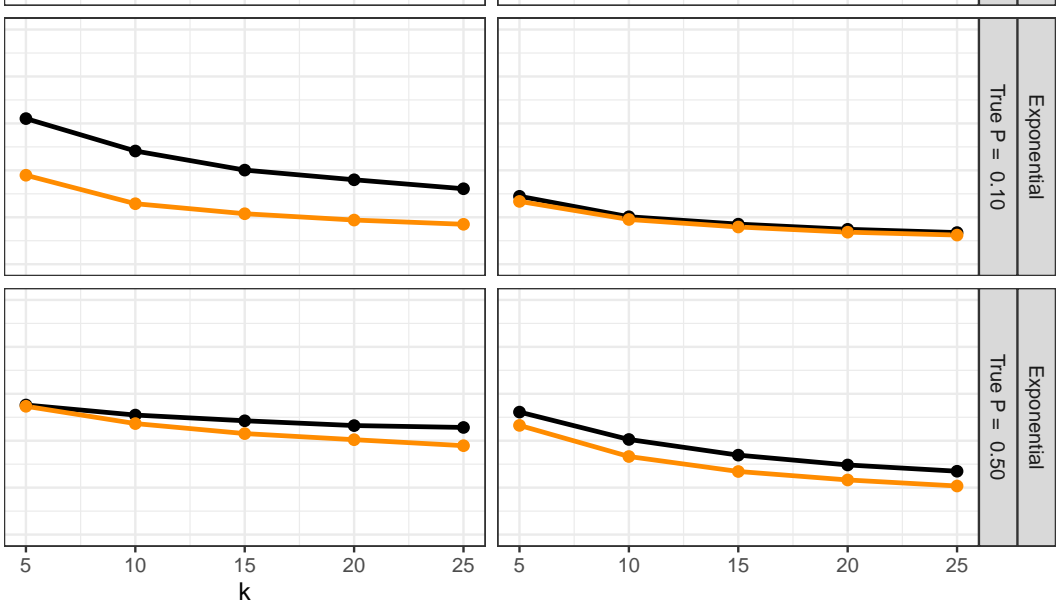

Figure S7: $R M S E$ of $\widehat{P}_{>q}$ for normal and exponential distributions 

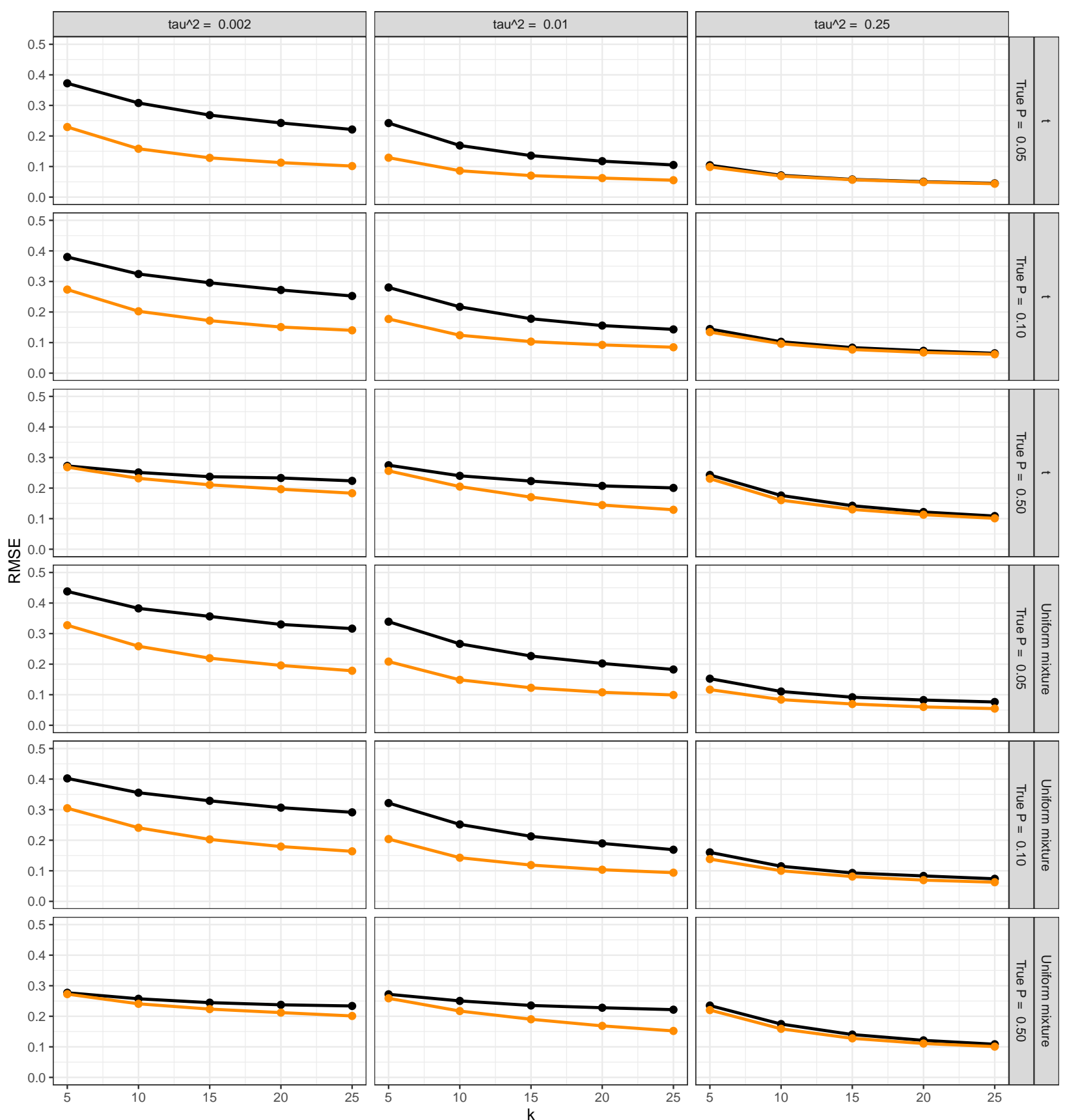

$\mathrm{N}_{\text {rep }}$
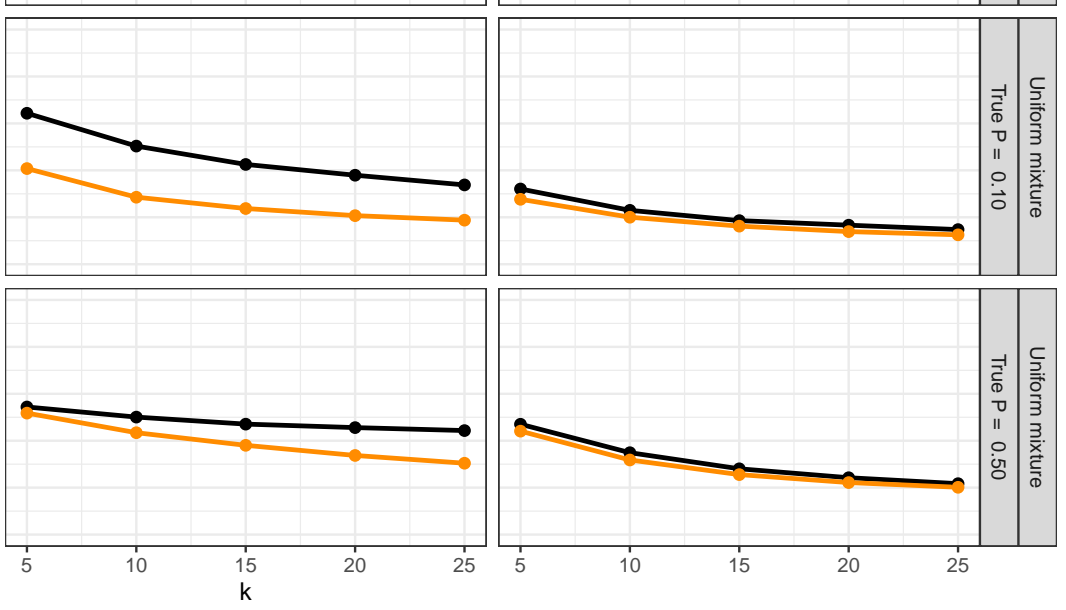

Figure S8: $R M S E$ of $\widehat{P}_{>q}$ for $t$ and uniform mixture distributions

\section{SOFTWARE}

The R package Replicate (version 1.2.0) contains the following functions; details are available in the standard $\mathrm{R}$ documentation. 
- prob_signif_agree computes the theoretical probability that a given replication study would agree in "statistical significance" and effect direction with the original study, if the true effect were indeed the same in the two studies.

- pred_int computes prediction interval limits and indicators for whether each replication estimate is within its corresponding prediction interval.

- p_orig computes $P_{\text {orig, }}$, i.e., the probability of observing an original estimate as extreme as that actually observed (compared to the replication studies) if the original were indeed consistent with the estimated distribution of the replication studies.

The R package MetaUtility (version 2.1.0) contains the following function, again with details in the standard $\mathrm{R}$ documentation.

- prop_stronger estimates $\widehat{P}_{>0}, \widehat{P}_{>q}$, or $\widehat{P}_{<q^{*}}$, i.e., the probability of a true effect above or below a user-specified threshold of scientific importance using estimates of the true effect distribution (based on the replications). 


\section{REFERENCES}

Braithwaite, R. S., Meltzer, D. O., King Jr, J. T., Leslie, D., \& Roberts, M. S. (2008). What does the value of modern medicine say about the $\$ 50,000$ per quality-adjusted life-year decision rule? Medical Care, 46(4), 349-356.

Chinn, S. (2000). A simple method for converting an odds ratio to effect size for use in meta-analysis. Statistics in Medicine, 19(22), 3127-3131.

Cohen, J. (1977). Statistical power analysis for the behavioral sciences. New York: Academic Press.

Crosby, R. D., Kolotkin, R. L., \& Williams, G. R. (2003). Defining clinically meaningful change in health-related quality of life. Journal of clinical epidemiology, 56(5), 395-407.

Eichler, H.-G., Kong, S. X., Gerth, W. C., Mavros, P., \& Jönsson, B. (2004). Use of cost-effectiveness analysis in health-care resource allocation decision-making: how are cost-effectiveness thresholds expected to emerge? Value in Health, 7(5), 518-528.

Hill, C. J., Bloom, H. S., Black, A. R., \& Lipsey, M. W. (2008). Empirical benchmarks for interpreting effect sizes in research. Child Development Perspectives, 2(3), 172-177.

Jaeschke, R., Singer, J., \& Guyatt, G. H. (1989). Measurement of health status: ascertaining the minimal clinically important difference. Controlled Clinical Trials, 10(4), 407-415.

Lakens, D., Scheel, A. M., \& Isager, P. M. (2018). Equivalence testing for psychological research: A tutorial. Advances in Methods and Practices in Psychological Science, 1(2), 259-269.

Monin, B., \& Miller, D. T. (2001). Moral credentials and the expression of prejudice. Journal of Personality and Social Psychology, 81(1), 33. 
Norman, G. R., Sloan, J. A., \& Wyrwich, K. W. (2003). Interpretation of changes in health-related quality of life: the remarkable universality of half a standard deviation. Medical Care, 41(5), 582-592.

Patil, P., Peng, R. D., \& Leek, J. T. (2016). What should researchers expect when they replicate studies? A statistical view of replicability in psychological science. Perspectives on Psychological Science, 11(4), 539-544.

Pettigrew, T. F., \& Tropp, L. R. (2008). How does intergroup contact reduce prejudice? meta-analytic tests of three mediators. European Journal of Social Psychology, 38(6), 922-934.

Redelmeier, D. A., Guyatt, G. H., \& Goldstein, R. S. (1996). Assessing the minimal important difference in symptoms: a comparison of two techniques. Journal of Clinical Epidemiology, $49(11), 1215-1219$.

Rücker, G., \& Schumacher, M. (2008). Simpson's Paradox visualized: the example of the rosiglitazone meta-analysis. BMC Medical Research Methodology, 8(1), 34 .

Stewart, G. B., Altman, D. G., Askie, L. M., Duley, L., Simmonds, M. C., \& Stewart, L. A. (2012). Statistical analysis of individual participant data meta-analyses: a comparison of methods and recommendations for practice. PloS One, 7(10), e46042.

VanderWeele, T. J. (2017). On a square-root transformation of the odds ratio for a common outcome. Epidemiology, 28(6), e58-e60. 